\title{
Dynamic Analysis, Circuit Design, and Synchronization of a Novel 6D Memristive Four-Wing Hyperchaotic System with Multiple Coexisting Attractors
}

\author{
Fei Yu $\left(\mathbb{D},{ }^{1}\right.$ Li Liu $\mathbb{D},{ }^{1}$ Hui Shen $\mathbb{D}^{1},{ }^{1}$ Zinan Zhang $\left(\mathbb{D},{ }^{1}\right.$ Yuanyuan Huang $\mathbb{D},{ }^{1}$ \\ Changqiong Shi $\mathbb{D}^{1},{ }^{1}$ Shuo Cai $\mathbb{D},{ }^{1}$ Xianming Wu $\mathbb{D}^{2},{ }^{2}$ Sichun Du $\mathbb{D}^{3},{ }^{3}$ and Qiuzhen Wan $\mathbb{D}^{4}$ \\ ${ }^{1}$ School of Computer and Communication Engineering, Changsha University of Science and Technology, Changsha 410114, China \\ ${ }^{2}$ School of Mechanical and Electrical Engineering, Guizhou Normal University, Guiyang 550025, China \\ ${ }^{3}$ College of Computer Science and Electronic Engineering, Hunan University, Changsha 410082, China \\ ${ }^{4}$ College of Information Science and Engineering, Hunan Normal University, Changsha 410081, China
}

Correspondence should be addressed to Fei Yu; yufeiyfyf@csust.edu.cn and Qiuzhen Wan; wanqiuzhen@sina.com

Received 28 February 2020; Revised 1 April 2020; Accepted 30 April 2020; Published 19 May 2020

Academic Editor: Lingzhong Guo

Copyright $\odot 2020$ Fei Yu et al. This is an open access article distributed under the Creative Commons Attribution License, which permits unrestricted use, distribution, and reproduction in any medium, provided the original work is properly cited.

In this work, a novel 6D four-wing hyperchaotic system with a line equilibrium based on a flux-controlled memristor model is proposed. The novel system is inspired from an existing 5D four-wing hyperchaotic system introduced by Zarei (2015). Fundamental properties of the novel system are discussed, and its complex behaviors are characterized using phase portraits, Lyapunov exponential spectrum, bifurcation diagram, and spectral entropy. When a suitable set of parameters are chosen, the system exhibits a rich repertoire of dynamic behaviors including double-period bifurcation of the quasiperiod, a single two-wing, and four-wing chaotic attractors. Further analysis of the novel system shows that the multiple coexisting attractors can be observed with different system parameter values and initial values. Moreover, the feasibility of the proposed mathematical model is also presented by using Multisim simulations based on an electronic analog of the model. Finally, the active control method is used to design the appropriate controller to realize the synchronization between the proposed 6D memristive hyperchaotic system and the $6 \mathrm{D}$ hyperchaotic Yang system with different structures. The Routh-Hurwitz criterion is used to prove the rationality of the controller, and the feasibility and effectiveness of the proposed synchronization method are proved by numerical simulations.

\section{Introduction}

Since the 1960s, nonlinear science has developed rapidly in various branches of disciplines. The in-depth study of nonlinear science not only has important theoretical value to the academic community, but also has a broad prospect for the practical application in life [1]. Chaos is one of the most important subjects in nonlinear motion, which creates a new situation of nonlinear science. Since the discovery of chaotic motion, chaotic dynamics has made rapid progress, and scientists from all over the world have made in-depth analysis and research on the characteristics of chaos [2-7]. Chaotic motion is a random behavior occurring in a defined nonlinear system. It is highly sensitive to initial conditions, has complex dynamic properties, and is difficult to predict. At present, it is widely used in complex networks [8-11], electronic circuits [12-15], image processing [16-20], random number generator [21-23], secure communication $[24,25]$, and other engineering fields.

For the application of chaos in engineering, it is sometimes a key problem to generate a chaotic attractor with a complex topological structure. Most research in this field has been focused on the multiwing attractors [26-28], multiscroll attractors [29-32], and chaotic systems in the fractional-order form [33-35]. More and more articles are written on this topic every day, and numerous articles are devoted to explain the new high-dimensional chaotic systems and more complicated topological structure. 
Compared with chaotic systems, hyperchaotic systems have more complex dynamic behaviors, which have two or more positive Lyapunov indices, more complex topological structures, and more unpredictable dynamic behaviors and are more difficult to crack. The most common method to construct hyperchaotic systems is to introduce new variables to the proposed chaotic systems to increase the dimensions of the differential equations and increase the nonlinear terms. Since the discovery of a first 4D hyperchaotic system by Rossler in 1979 [36], many 4D hyperchaotic systems have been found in the literature such as hyperchaotic Lorenz system [37], hyperchaotic Chen system [38], hyperchaotic Lü system [39], hyperchaotic Yu system [40], hyperchaotic Wang system [41], and hyperchaotic Vaidyanathan system [42]. Recently, people have developed a strong interest in searching for $5 \mathrm{D}$ and $6 \mathrm{D}$ hyperchaotic systems with more complex dynamic behavior and such 5D and 6D hyperchaotic systems have been found in the literature such as hyperchaotic Vaidyanathan system [43], hyperchaotic Kemih system [44], hyperchaotic Lorenz system [45], and hyperchaotic Yang system [46]. Hyperchaotic systems can also produce multiscroll or multiwing attractors, which is a very important phenomenon. In recent years, some fourwing hyperchaotic attractors have appeared [47, 48]. These attractors generally have five equilibrium points, and each wing hovers near a nonzero equilibrium point. The three or five equilibrium points of the chaotic system are very important, especially in the multiscroll or multiwing chaotic system, but the multiscroll or multiwing hyperchaotic attractor with a linear equilibrium point is exciting.

Memristor is a nonlinear passive element with nonlinearity and nonvolatility. In recent years, the research work has made gratifying progress, and the application of various memristors has become a research hotspot [49-51]. In 2008, scientists at HP LABS successfully built the first physically realized memristor [52], confirming the prediction of professor Chua in 1971 [53]. Since then, memristors have received extensive attention and research. Due to its small size and low power consumption, a memristor is an ideal choice for nonlinear circuits in chaos [54]. The common methods to produce hyperchaos are the linear feedback method and the nonlinear feedback method. Among them, the nonlinear feedback method is better than the linear feedback method. However, the product term of the nonlinear function makes the realization circuit more complex. If the memristor is used as the nonlinear feedback, it will greatly reduce the difficulty of circuit realization. At the same time, the memory ability of a memristor to flow through current is not possessed by conventional chaotic circuit elements [55]. Therefore, it is of practical significance to study the application of a memristor in a hyperchaotic system, and various hyperchaotic systems based on memristors have been paid close attention by researchers [56-59].

In order to construct memristive hyperchaotic systems with more complex dynamics, some kind of $5 \mathrm{D}$ and $6 \mathrm{D}$ memristive hyperchaotic systems have been proposed recently [60-62]. In [60], a novel 5D hyperchaotic four-wing memristive system (HFWMS) was proposed by introducing a flux-controlled memristor with quadratic nonlinearity into a 4D hyperchaotic system as a feedback term. The HFWMS with multiline equilibrium and three positive Lyapunov exponents presented very complex dynamic characteristics, such as the existence of chaos, hyperchaos, limit cycles, and periods. In [62], a 6D autonomous system was presented by introducing a flux-controlled memristor model into an existing 5D hyperchaotic autonomous system, which exhibited hyperchaotic under a line or a plane of equilibria. Some other attractive dynamics were also observed, like hidden extreme multistability, transient chaos, bursting, and offset boosting phenomenon. It can be seen that such superhigh-dimensional attractors cannot be ignored. Because of their complexity, the generated signals are usually suitable for secure communication and random number generation, so the super-high-dimensional attractors will be an added value to their randomness.

Coexistence of multiple attractors is a kind of singular physical phenomenon often encountered in a nonlinear dynamic system. Under the condition of constant system parameters, when the initial state is changed, the trajectory of the system may asymptotically approach different stable states such as trend point, chaos, period, and quasiperiod $[15,23,46]$. In some special coupling systems and novel memristive chaotic systems, the coexistence of infinite number of attractors can also be observed [62]. Common multiple coexisting attractors generally have symmetry, and there is symmetric coexistence of left and right or upper and lower attractors. Recently, it has been found that the coexistence of asymmetric multiattractors also exists in some special systems, which is a new nonlinear phenomenon $[61,62]$. Multiple coexisting attractors provide a great degree of freedom for the engineering application of nonlinear dynamic systems and also present a new challenge to the multistability state switching control technology. Therefore, the study of multiple coexisting attractors and their synchronization has important theoretical physical significance and engineering application value.

With the rapid development of network communication technology, the confidentiality of information and the security of the system is not considered complete, resulting in increasingly serious information security problems. Information security technology mainly includes monitoring, scanning, detection, encryption, authentication, and attack prevention [63-72]. Due to the characteristics of chaotic systems such as aperiodic, continuous wideband, and noiselike, the use of chaotic synchronization has more stringent communication confidentiality, so it has received great attention in the field of information security. Pecora and Carroll [73] first proposed the concept of chaotic synchronization in 1990 and observed the phenomenon of chaotic synchronization on electronic circuits. This pioneering work greatly promoted the study of chaotic synchronization theory. Since then, complete synchronization [74], antisynchronization [40], generalized synchronization [75], projection synchronization [76, 77], lag synchronization [78], function projection synchronization [79], and shape synchronization [80] methods have been widely studied in the literature. 
In this paper, a novel $6 \mathrm{D}$ memristive hyperchaotic system is proposed based on a flux-controlled memristor model and the 5D hyperchaotic system introduced in [48]. Most importantly, the novel system generates the striking phenomenon of multiple coexisting chaotic attractors and exhibits hyperchaos with a line equilibrium. Under certain parameters and initial conditions, the system exits doubleperiod bifurcation of the quasiperiod, which can produce four-wing hyperchaotic and chaotic attractors. A notable feature of the new system is the ability to generate two-wing and four-wing smooth chaotic attractors with special appearance. Then, an electronic circuit realization of the novel 6D memristive four-wing hyperchaotic system is presented to confirm the feasibility of the theoretical model. Finally, an adaptive active controller is designed to realize the global hyperchaos synchronization of the novel $6 \mathrm{D}$ memristive four-wing hyperchaotic systems and the 6D Yang hyperchaotic system with different structures.

The rest of this work is structured as follows: In Section 2, the mathematical model of the novel 6D memristive hyperchaotic system that can generate two-wing and fourwing attractors is introduced. Numerical findings of the novel 6D memristive hyperchaotic system are carried out in Section 3 by using classical nonlinear diagnostic tools. The multiple coexisting attractors of the system are investigated, and the spectral entropy complexity is also reported. Some Multisim simulations based on a suitable designed electronic analog circuit diagram of the model are carried out to show its feasibility in Section 4. In Section 5, the novel chaotic system's active control synchronization is derived. Finally, Section 6 draws the concluding remarks of this work.

\section{A Novel 6D Memristive Four-Wing Hyperchaotic System}

Recently, Zarei [48] proposed a 5D hyperchaotic system, whose differential equation can be described as

$$
\left\{\begin{array}{l}
\dot{x}=-a x+y z \\
\dot{y}=-b y+f u \\
\dot{z}=-c z+x y+g w \\
\dot{w}=d w-h z \\
\dot{u}=e u-x^{2} y
\end{array}\right.
$$

where $x, y, z, w$, and $u$ are the state variables of the system and $a, b, c, d, e, f, g$, and $h$ are the system parameters. The system has many interesting complex dynamical behaviors such as periodic orbit, chaos, and hyperchaos with only one equilibrium point. When proper system parameters and initial values are selected, the system can exhibit four-wing hyperchaotic attractors. The system has been well studied in [48], which shows the coexistence attractor and hyperchaotic attractor of two positive Lyapunov exponents. However, memristor chaos is not part of this feature. Our goal is to construct a high-dimensional system with coexistence attractors and memristor, thus forming a system of ordinary differential equations of memristive four-wing high-dimensional hyperchaos.
Memristor is a passive two-terminal device that describes the relationship between magnetic flux $\varphi$ and charge $q$. The memristor used in this work is a flux-controlled memristor, which is described by the nonlinear constitutive relation between the terminal voltage $u$ and the terminal current $i$ of the device, i.e.,

$$
i=W(\varphi) u, \dot{\varphi}=u
$$

where $W(\varphi)$ is a memductance function which is called the incremental memductance, defined as $W(\varphi) \equiv d q(\varphi) / \varphi$.

In this paper, the $\varphi-q$ characteristic curve of the memristor is given by a smooth continuous cubic monotone-increasing nonlinearity, i.e., $q(\varphi)=m+n \varphi^{3}$, where $m, n>0$. Thus, the memductance in this paper is given by

$$
W(\varphi)=m+3 n \varphi^{2} \text {. }
$$

By introducing the lux-controlled memristor model (3) into the second equation of system (1), a novel 6D memristive autonomous hyperchaotic system is constructed

$$
\left\{\begin{array}{l}
\dot{x}=-a x+y z \\
\dot{y}=-b y+f\left(m+3 n \varphi^{2}\right) u \\
\dot{z}=-c z+x y+g w \\
\dot{w}=d w-h z \\
\dot{u}=e u-x^{2} y \\
\dot{\varphi}=u
\end{array}\right.
$$

where $x, y, z, w, u$, and $\varphi$ are the state variables; $a, b, c, d, e, f, g, h, m$, and $n$ are the system parameters. When $a=10, b=60, c=20, d=15, e=40, f=1, g=50$, $h=10, m=1,3 n=0.02$, and the initial condition is set to $[1,1,1,1,1,1]$, we use the Runge-Kutta algorithm (RK45) to solve the differential equation. Figure 1 shows the phase portraits of system (4) obtained through MATLAB simulation. It can be seen from the figure that the proposed system presents four-wing chaos in different phase planes.

In general, symmetry is widespread in chaotic systems, and system (4) is invariant under the coordinate transformation $(x, y, z, w, u, \varphi) \longrightarrow(-x,-y, z, w,-u,-\varphi)$ and has the same symmetry as the original 5D system (1).

Let the six equations at the right end of system (4) be zero, and the equilibrium point of system (4) can be obtained by solving the following equations:

$$
\left\{\begin{array}{l}
-a x+y z=0 \\
-b y+f\left(m+3 n \varphi^{2}\right) u=0 \\
-c z+x y+g w=0 \\
d w-h z=0 \\
e u-x^{2} y=0 \\
u=0
\end{array}\right.
$$

According to equation (5), system (4) has a line equilibrium point $O=\{(x, y, z, w, u, \varphi) \mid x=y=z=w=0$, $u=0, \varphi=l\}$, which means that every point on the $\varphi$-axis is the system equilibrium point, where $l$ is an arbitrary real 


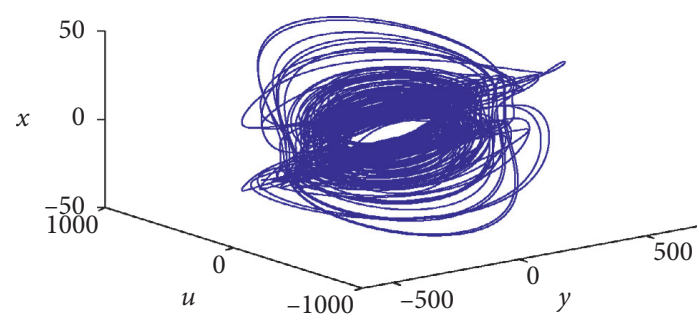

(a)

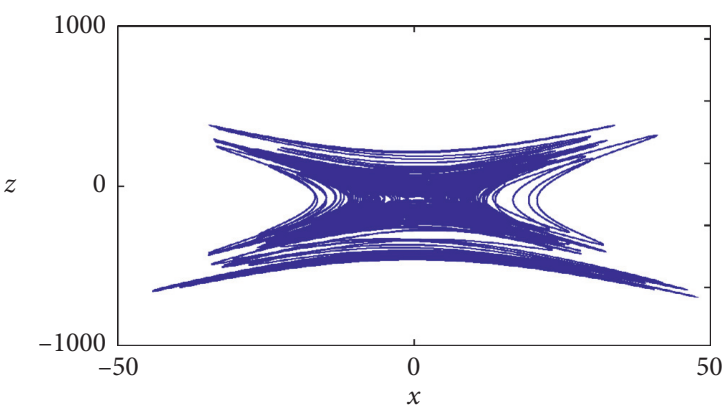

(c)

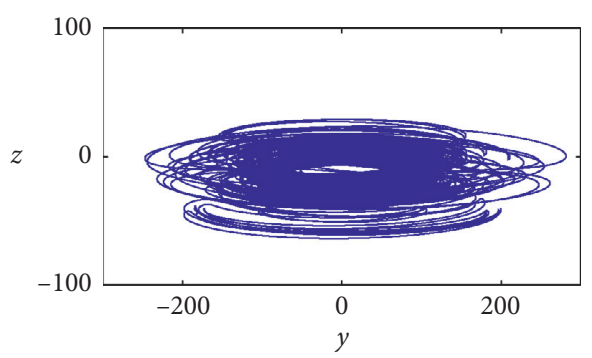

(b)

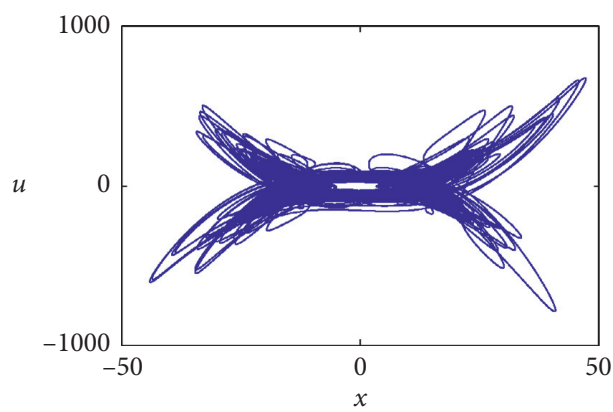

(d)

Figure 1: The four-wing chaotic attractor of system (4) in the (a) $y-u-x$ plane, (b) $y-z$ plane, (c) $x-z$ plane, and (d) time-domain waveform of $x-u$.

constant. The Jacobian matrix at the line equilibrium point $O$ of system (4) is

$$
J_{o}=\left[\begin{array}{cccccc}
-a & z & y & 0 & 0 & 0 \\
0 & -b & 0 & 0 & f\left(m+3 n \varphi^{2}\right) & 6 f n \varphi u \\
y & x & -c & g & 0 & 0 \\
0 & 0 & -h & d & 0 & 0 \\
-2 x y & -x^{2} & 0 & 0 & e & 0 \\
0 & 0 & 0 & 0 & 1 & 0
\end{array}\right] .
$$

According to (6), the characteristic equation can be obtained:

$$
\lambda(\lambda-e)(\lambda+a)(\lambda+b)\left(\lambda-m_{1}\right)\left(\lambda-m_{2}\right)=0,
$$

where

$$
\begin{aligned}
& m_{1}=\frac{(d-c)+\sqrt{(c-d)^{2}-4(g h-c d)}}{2}, \\
& m_{2}=\frac{(d-c)-\sqrt{(c-d)^{2}-4(g h-c d)}}{2} .
\end{aligned}
$$

According to the characteristic equation and system parameters, $\lambda_{1}=0, \lambda_{2}=40, \lambda_{3}=-10, \lambda_{4}=-60, \lambda_{5}=-2.5+$ $13.9194 i$, and $\lambda_{6}=-2.5-13.9194 i$ can be obtained. Therefore, there are one positive eigenvalue, one zero eigenvalue, and two negative eigenvalues, and the line equilibrium of system (4) is unstable saddle points.

The divergence of system (4) is given by

$$
\nabla V=\frac{d \dot{x}}{d x}+\frac{d \dot{y}}{d y}+\frac{d \dot{z}}{d z}+\frac{d \dot{w}}{d w}+\frac{d \dot{u}}{d u}+\frac{d \dot{\varphi}}{d \varphi}=-a-b-c+d+e
$$

since $-a+b-c-e=-35$ satisfies $\nabla V<0$, system (4) is dissipative and converges exponentially.

\section{Dynamic Analysis of the Novel 6D Memristive Chaotic System}

In this section, with the help of a bifurcation diagram, Lyapunov exponent spectrum, and phase portraits, we will use the fourth-order Runge-Kutta algorithm to numerically study the complex dynamic behavior of system (4) by MATLAB.

3.1. Fix Other Parameters and Change Parameter a. Given parameters $b=60, c=20, d=15, e=40, f=1, g=50, h=$ $10, m=1$, and $3 n=0.02$ and initial conditions $(0)=1$, $y(0)=1, \quad z(0)=1, w(0)=1, u(0)=1$, and $\varphi(0)=1$, let parameter $a$ be the bifurcation parameter of system (4), where Figure 2(a) shows the bifurcation diagram when system parameter $a$ changes from 0 to 12 , and Figure 2(b) shows the corresponding Lyapunov exponent spectrum. It can be seen from Figure 2 that the system is chaotic in $[0,4.6]$ and hyperchaotic in $(4.6,12]$. When $a=12$, the value of the Lyapunov exponent is 12.56 , which is the maximum value of the simulation interval and larger than the maximum Lyapunov exponent of system (1) $\left(\mathrm{LE}_{\max }=9.979\right)$. Suffice it to say, the introduction of a memristor can make the system more complex. When $a=10$, we use the famous wolf method to calculate the 


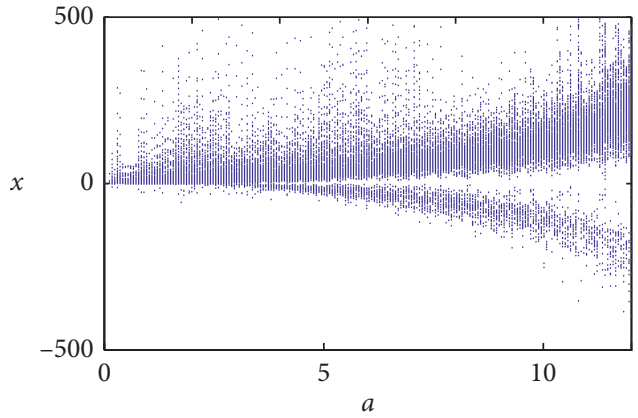

(a)

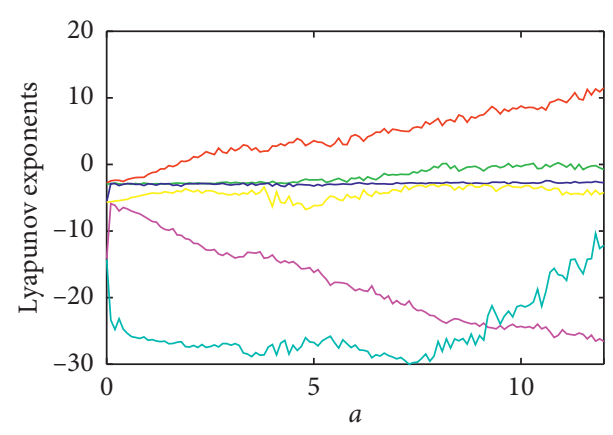

(b)

FIGURE 2: Lyapunov exponent spectrum and bifurcation diagram for parameter $a \in[0,12]$ : (a) bifurcation diagram; (b) Lyapunov exponent spectrum.

Lyapunov exponents. The LEs are $\mathrm{LE}_{1}=10.16, \mathrm{LE}_{2}=2.187$, $\mathrm{LE}_{3}=0.0136, \mathrm{LE}_{4}=-0.5759, \mathrm{LE}_{5}=-16.08$, and $\mathrm{LE}_{6}=-18.86$. There are two positive Lyapunov exponents, so system (4) is hyperchaotic. Based on the Lyapunov exponents, we also get the Kaplan-Yorke dimension that describes the complexity of the attractor. It can be computed by

$$
D_{\mathrm{KY}}=D+\sum_{i=1}^{D} \frac{\mathrm{LE}_{i}}{\left|\mathrm{LE}_{D}\right|}
$$

where $D$ is a constant satisfying $\sum_{i=1}^{D} \mathrm{LE}_{i} \geq 0$ and $\sum_{i=1}^{D+1} \mathrm{LE}_{i}<0$. According to equation (10), the Kaplan-Yorke dimension of system (4) is 4.7723 , so the attractors generated by the new system are strange attractors.

3.2. Fix Other Parameters and Change Parameter $d$. Given parameters $a=10, b=60, c=20, e=40, f=1$, $g=50, h=10, m=1$, and $3 n=0.02$ and initial conditions $(0)=1, y(0)=1, \quad z(0)=1, w(0)=1, u(0)=1$, and $\varphi(0)=$ 1 , when parameter $d \in[-10,20]$, Figure $3(\mathrm{a})$ shows the bifurcation diagram changing with parameter $d$, and Figure 3(b) shows the corresponding Lyapunov exponent spectrum. It can be seen from Figure 3 that the system has doubly periodic bifurcation, chaos, and hyperchaos phenomena. The double-period bifurcation simulated in this paper is different from the simulation results of most papers, which are double-period bifurcation of the period, while in this paper, it is the double-period bifurcation of the quasiperiod. Table 1 gives a summary of dynamic characteristics of parameter $d$. The following analysis shows the dynamic behavior with respect to parameter $d$ :

(i) When $d=-2$, the maximum Lyapunov exponent of system (4) is zero $\left(\mathrm{LE}_{1,2}=0, \mathrm{LE}_{3,4,5,6}<0\right)$, and the system is in a quasiperiodic 1 state. Figure 4(a) shows the corresponding phase portraits;

(ii) When $d=-1$, the maximum Lyapunov exponent of system (4) is zero $\left(\mathrm{LE}_{1,2}=0, \mathrm{LE}_{3,4,5,6}<0\right)$, and the system is in a quasiperiodic 2 state. Figure 4(b) shows the corresponding phase portraits;

(iii) When $d=0$, system (4) has a positive Lyapunov exponent $\left(\mathrm{LE}_{1}>0, \mathrm{LE}_{2}=0, \mathrm{LE}_{3,4,5,6}<0\right)$, and the system behaves as a two-wing chaotic attractor state. The corresponding phase portrait is shown in Figure 4(c);

(iv) When $d=16$, system (4) has two positive Lyapunov exponents $\left(\mathrm{LE}_{1,2}>0, \mathrm{LE}_{3}=0\right.$, and $\left.\mathrm{LE}_{4,5,6}<0\right)$, and the system is in a four-wing hyperchaos state. The corresponding four-wing phase portrait is shown in Figure 4(d).

3.3. Multiple Coexisting Attractors. In this section, we will study the multiple coexisting attractors of the proposed $6 \mathrm{D}$ memristive hyperchaotic system. Fixed system parameters are $a=10, b=60, c=20, e=40, f=1, g=50, h=10, m=$ 1 , and $3 n=0.02$. When $d=-3$ and $d=-0.5$, two different initial conditions $[1,1,1,1,1,1]$ and $[1,1,-1,1,1,1]$ are taken to observe the phenomenon of coexistence quasiperiodic 1 and coexistence quasiperiodic 2 as shown in Figures 5(a) and 5(b). When $d=0$, two different initial conditions $[1,1,1,1,1,1]$ and $[-1,1,1,1,1,1]$ are taken to observe the coexistence of two-wing chaotic attractors presented in Figure 5(c). Choosing $d=15$ and taking two different initial conditions $[1,1,0.001,1,1,1]$ and $[1,1,-0.001,1,1,1]$, the coexistence of four-wing hyperchaotic attractors is observed in Figure 5(d). When $d=-7$ is selected, the initial conditions $[1,1,1,1,1,1]$, $[-1,1,1,1,1,1],[20,1,1,1,1,1]$, and $[-20,1,1,1,1,1]$ are selected, as shown in Figure 5(e); there are four quasiperiodic attractors coexisting, and the four attractors are symmetric.

When the system parameters are selected as $a=1, b=$ $8, c=1, d=-20, e=1, f=2, g=1, h=-1, m=1$, and $3 n=$ 0.02 , the phase portraits of system (4) under different initial conditions are shown in Figure 6. Figure 6(a) shows the coexistence of four one-wing period- 1 attractors, Figure 6(b) shows the coexistence of four one-wing multiperiod attractors, Figure 6(c) shows the coexistence of two-wing multiperiod attractors, and Figure 6(d) shows the coexistence of four two-wing multiperiod attractors. When the system parameters are selected as $a=1, b=5$, $c=1, d=-20, e=1, f=2, g=1, \quad h=-1, m=1$, and $3 n=$ 0.02 , the phase portraits of system (4) under different initial conditions are shown in Figure 7. In Figure 7(a), two-wing 


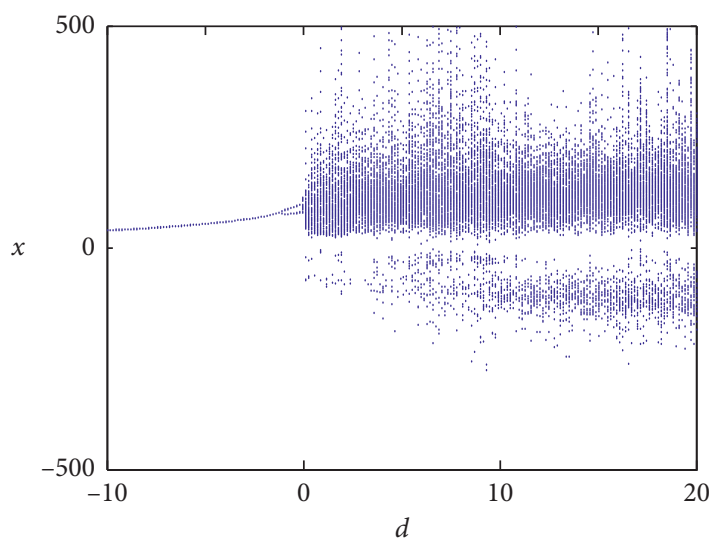

(a)

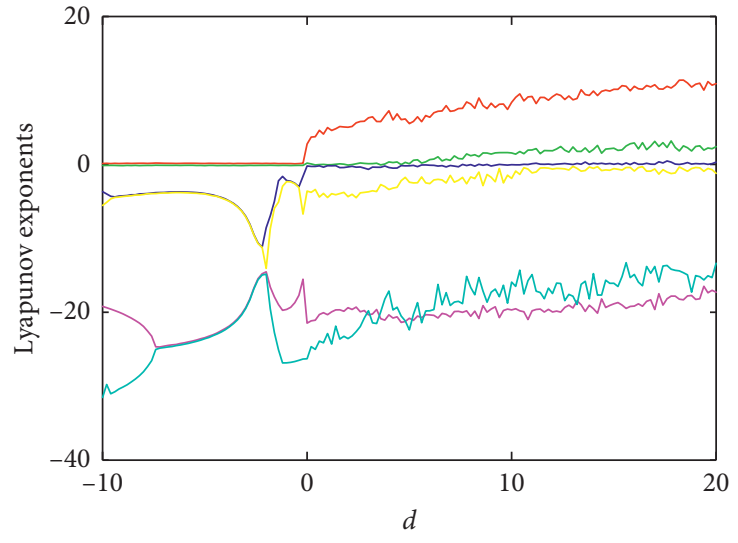

(b)

FIGURE 3: Lyapunov exponent spectrum and bifurcation diagram for parameter $d \in[-10,20]$ : (a) bifurcation diagram; (b) Lyapunov exponent spectrum.

TABle 1: Dynamical behavior and Lyapunov exponents under different parameter range of $d$.

\begin{tabular}{lccc}
\hline$d$ & $\left(\mathrm{LE}_{1}, \mathrm{LE}_{2}, \mathrm{LE}_{3}, \mathrm{LE}_{4}, \mathrm{LE}_{5}, \mathrm{LE}_{6}\right)$ & Dynamic & Figure \\
\hline$[-10,-1.2]$ & $(0,0,-,-,-,-)$ & Quasiperiodic 1 & Figure 4(a) \\
$(-1.2,0)$ & $(0,0,-,-,-,-)$ & Quasiperiodic 2 & Figure 4(b) \\
{$[0,5]$} & $(+, 0,-,-,-,-)$ & Chaotic & Figure 4(c) \\
$(5,20]$ & $(+,+, 0,-,-,-)$ & Hyperchaotic & Figure 4(d) \\
\hline
\end{tabular}

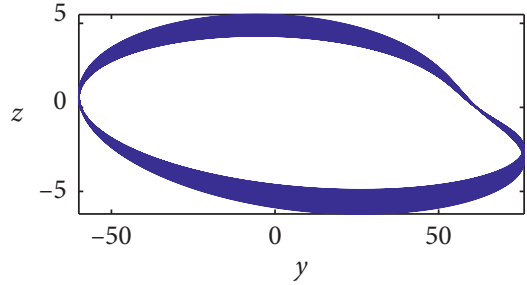

(a)

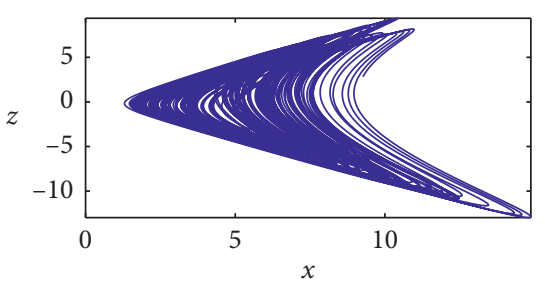

(c)

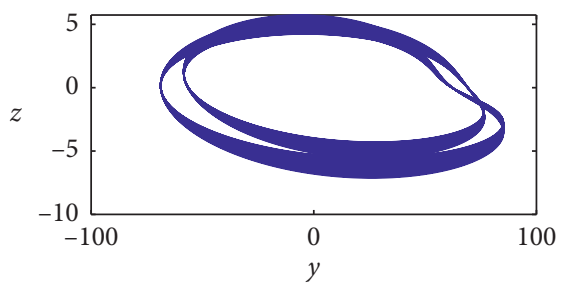

(b)

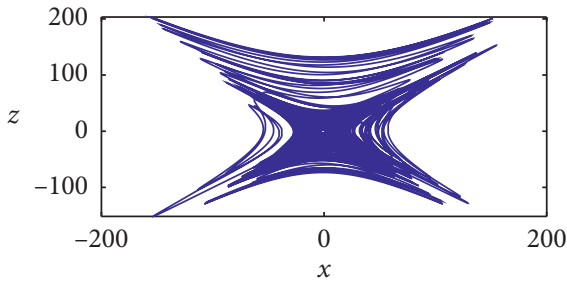

(d)

FIgURE 4: The phase portraits: (a) quasiperiodic 1, (b) quasiperiodic 2, (c) two-wing chaotic attractor, and (d) four-wing hyperchaotic attractor.

period-1 attractors coexist; in Figure 7(b), two-wing period1 attractors coexist; in Figure 7(c), four-wing period-1 attractors coexist; in Figure $7(\mathrm{~d})$, two-wing multiperiod attractors coexist, among which cyan, red, yellow, and earthy yellow are one group; black, green, blue, and magenta are the other. Figure 8 shows the phase portraits of different attractors when the initial conditions are $[1,1,1,1,1,1]$ and $[1,1,-1,1,1,1]$, but the parameter values in Figures $8(\mathrm{a})$ and 8 (b) are different. The parameter values in Figure $8(a)$ are fixed to $a=2, b=6, c=1, d=-20, e=1, f=2, g=1, h=$ $-1, m=1$, and $3 n=0.02$. It can be seen from the figure that the system has the coexistence of two-wing chaotic attractors. The parameter values in Figure 8(b) are fixed to $a=$ $2, b=6, c=2, d=-2, e=2, f=2, \quad g=-1, h=-1, m=1$, and $3 n=0.02$. It can be seen from the Figure that the limit cycle presented by the system is completely symmetric. In conclusion, the attractors generated by the new system are symmetric with respect to different initial conditions. 


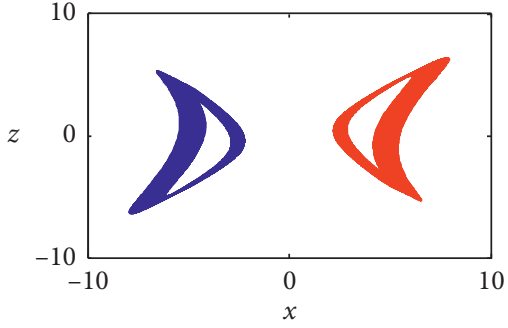

(a)

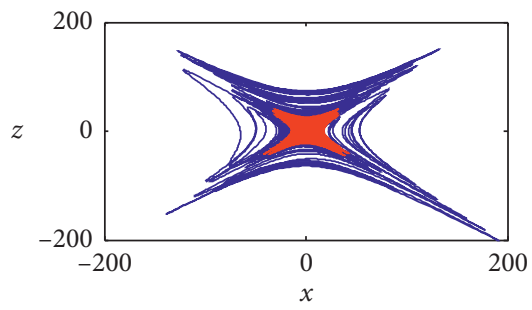

(d)

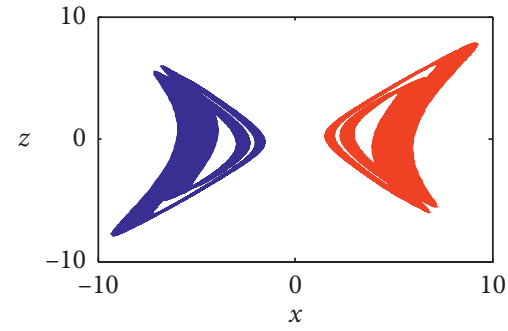

(b)

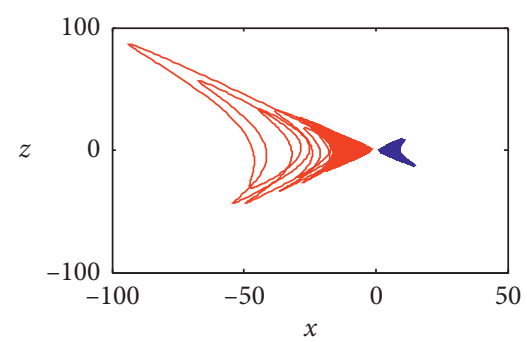

(c)

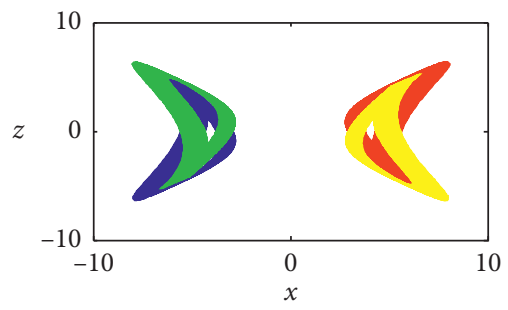

(e)

Figure 5: Various coexisting attractors with different values of parameter $d$ in the $x-z$ plane: (a) $d=-3$, (b) $d=-0.5$, (c) $d=0$, (d) $d=15$, and (e) $d=-7$.

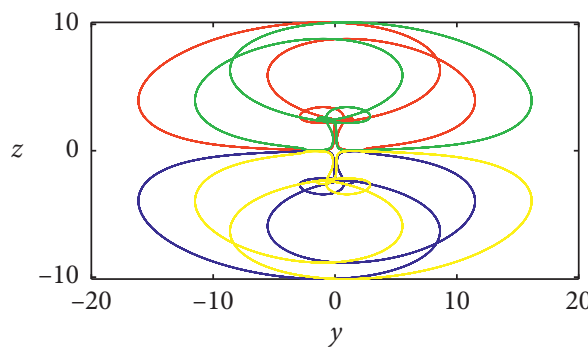

(a)

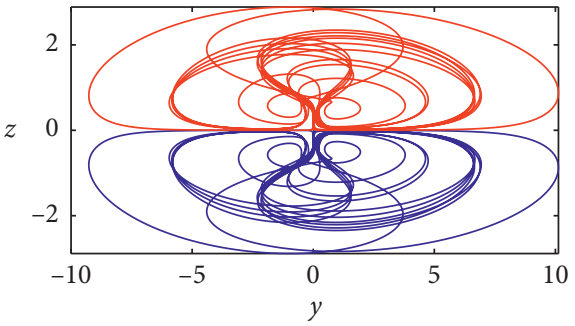

(c)

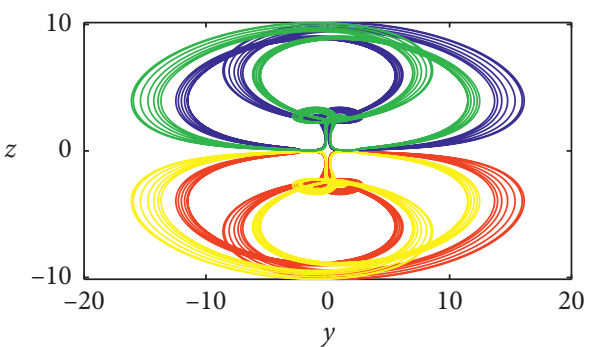

(b)

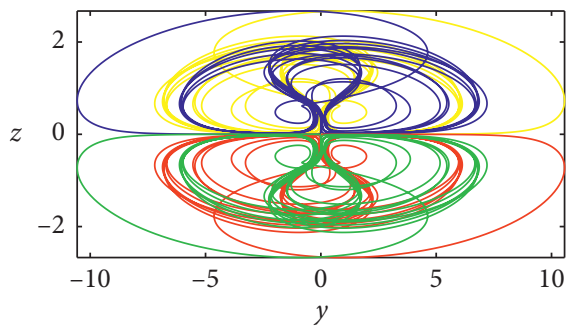

(d)

Figure 6: Coexisting attractors in the memristive hyperchaotic system: projections of different attractors on the $y-z$ plane for different initial conditions. (a) Symmetric period-1, $[1,1,1,1,1,1]$ (red), $[-1,-1,-1,1,1,1]$ (blue), $[-1,-1,1,1,-1,-1]$ (green), and $[1,1,-1,-1,-1,1]$ (yellow). (b) Symmetric one-wing multiperiod, $[-1,1,1,-1,1,1]$ (red), $[1,1,-1,1,1,1]($ blue), $[-1,-1,-1,1,-1,-1]$ (green), and $[1,-1,1,-1,-1,-1]$ (yellow). (c) Symmetric two-wing multiperiod, $[-1,1,1,1,1,1]$ (red) and $[1,1,-1,-1,1,1]$ (blue). (d) Symmetric two-wing multiperiod, $[-1,1,-1,1,-1,-1]$ (red), $[-1,-1,1,-1,1,1]$ (blue), $[1,-1,-1,1,1,1]$ (green), and $[1,1,1,-1,-1,-1]$ (yellow).

3.4. Complexity Analysis of Spectral Entropy. Spectral entropy (SE) algorithm is based on the Fourier transform to calculate the relative power spectrum and the Shannon entropy to calculate the SE complexity of the sequence, which reflects the disorder of time series in the frequency domain [81]. If the spectrum of the sequence is more complex, the SE of the chaotic system will be larger, making the system more complex, otherwise the system complexity is low [82]. Generally, the SE algorithm can be described as follows: given a chaotic random sequence $\{x(n), n=0,1,2, \ldots, N-1\}$ of length $N, x(n)=x(n)-\bar{x}$ is adopted to remove the $\mathrm{dc}$ part, where $\bar{x}$ is the mean value of the given sequence, and discrete Fourier transform is performed on sequence $x(n)$ : 


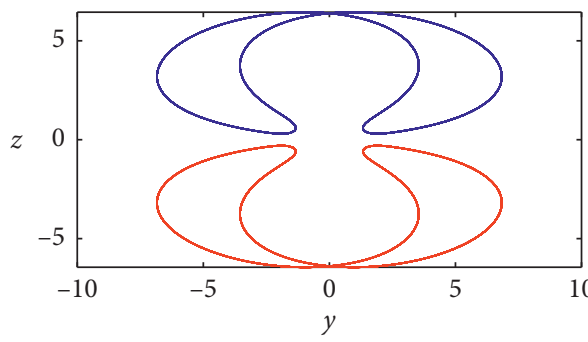

(a)

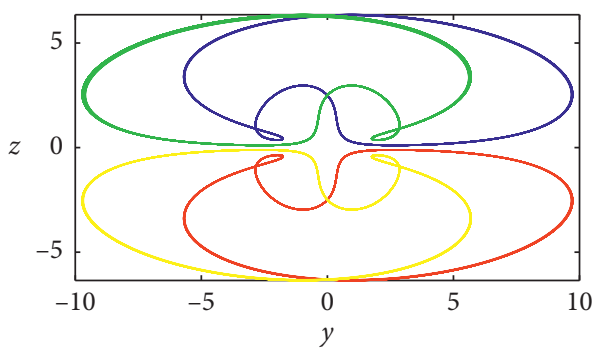

(c)

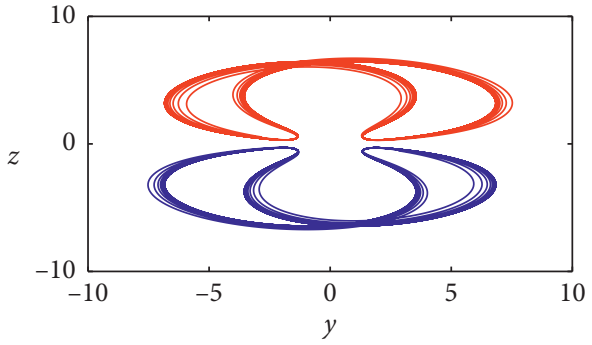

(b)

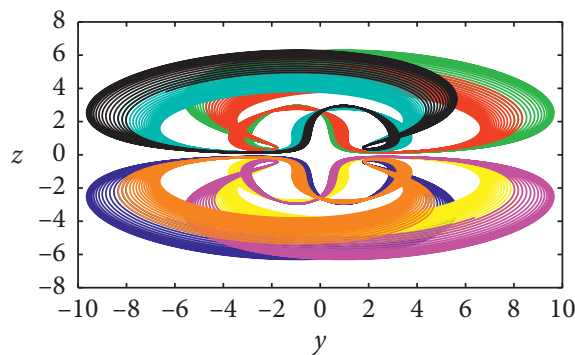

(d)

FiguRE 7: Coexisting attractors in the memristive hyperchaotic system: projections of different attractors on the $y-z$ plane for different initial conditions. (a) Symmetric two-wing period-1, $[1,1,1,1,1,1]$ (blue) and $[1,-1,-1,1,-1,1]$ (red). (b) Symmetric two-wing multiperiod, $[-1,1,1,1,1,-1]$ (red) and $[-1,-1,-1,-1,-1,1]$ (blue). (c) Symmetric one-wing period-1, [-1,-1,-1,-1,-1,-1] (red), $[-1,1,1,1,1,1]$ (blue), $[1,1,1,-1,-1,1]$ (green), and $[-1,1,-1,-1,-1,-1]$ (yellow). (d) Symmetric one-wing multiperiod, $[-1,-1,1,-1,1,1]$ (red), $[1,-1,1,-1,-1,-1]$ (blue), [1, 1, -1, 1, 1, 1] (green), [1,-1,-1,1,1,1] (yellow), [-1, -1, -1, 1, -1, -1] (black), $[-1,1,-1,1,-1,-1]$ (khaki), $[-1,1,1,-1,1,1]$ (magenta), and $[1,1,1,-1,-1,-1]$ (cyan).

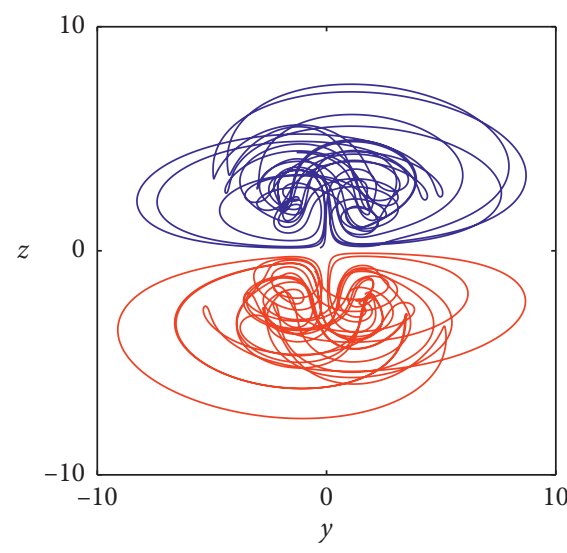

(a)

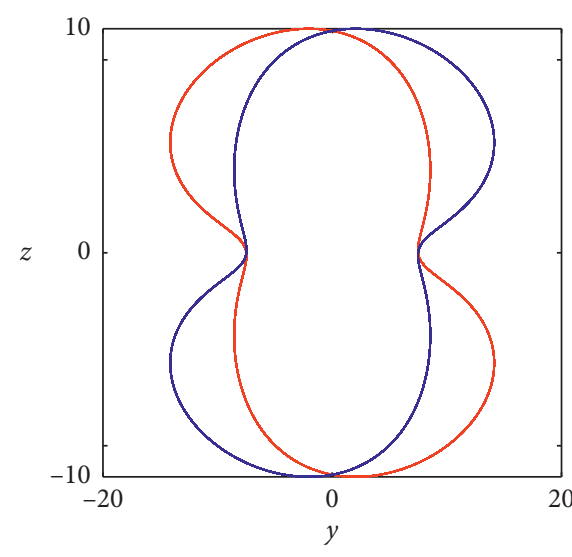

(b)

Figure 8: Various coexisting attractors in the $y-z$ plane under initial conditions $[1,1, \pm 1,1,1,1]$ : (a) coexistence of two-wing chaotic attractors and (b) coexistence of limit cycles.

$$
\mathrm{X}(k)=\sum_{n=0}^{N-1} x(n) e^{-j 2 \pi n k / N}
$$

where $k=0,1,2, \ldots, N-1$. Taking half the total power of the calculation sequence for $X(k)$ :

$$
p_{t}=\frac{1}{N} \sum_{k=0}^{N / 2-1}|X(k)|^{2} .
$$

According to the total power of the sequence, the relative power spectrum probability of the sequence is obtained:

$$
p_{k}=\frac{|X(k)|^{2}}{\sum_{k=0}^{N / 2-1}|X(k)|^{2}} .
$$

The normalized SE is

$$
\mathrm{SE}=\frac{\mathrm{se}}{\ln (N / 2)},
$$

where se $=-\sum_{k=0}^{N / 2-1} P_{k} \ln P_{k}$. Using $p_{k}$ and the Shannon entropy, the spectral entropy of the system is obtained.

The complexity of system (4) is analyzed by the SE algorithm. The control parameters $a$ and $d$ of the chaotic system 
are divided into $101 \times 101$ parts, where $a \in[0,12]$ and $d \in[-10,20]$, and then the SE of each point $(a, d)$ in the parameter space is calculated. Figure 7 shows the SE diagram of system (4) based on the previous algorithm. It can be seen from the figure that Figures 9(a) and 9(b) well correspond to the largest Lyapunov exponents in Figures 2 and 3. The results show that with the increase of parameters $a$ and $d$, the higher the complexity of the chaotic system is, the higher the complexity of the system is mainly concentrated in $a \in[4.6,12]$ and $d \in(0,20]$. Figure 9(c) shows the SE complexity in control parameters $a$ and $d$ planes. It can be seen from the figure that the system has high complexity in a large range, which means chaos or hyperchaos in these ranges.

\section{Circuit Design}

In recent years, the implementation of a chaotic system by hardware mainly includes analog discrete component circuit, CMOS integrated circuit, and continuous chaotic signal by modern digital signal processing technology, such as FPGA. CMOS technology is used to realize the chaotic oscillator circuit, which has the characteristics of low power consumption and small area [12-14, 49], but the design needs a long period, high cost, and difficult tuning [83-85]. Because of its large capacity and high reliability, FPGA is widely used in modern digital signal processing. However, FPGA needs a discrete continuous system, writing the underlying hardware code and requiring the computational intensive reading $[15,21,60]$. It is the most common method to generate a chaotic signal by using discrete components to design an analog circuit with simple structure, low cost, and easy operation [26-28, 30-32, 57-59, 61]. To further verify the dynamic characteristics of system (4), the system circuit was designed using discrete components: resistors, capacitors, operational amplifiers, and multipliers. In the circuit design, LF347 is used as the operational amplifier, the multiplier is AD633JN, and the multiplication factor is $0.1 / \mathrm{V}$. The operating voltage of operational amplifier is $\pm E= \pm 15 \mathrm{~V}$, and the saturation voltage measured by the operational amplifier and the multiplier is $\pm|V s a t| \approx \pm 13.5 \mathrm{~V}$. The relevant circuit equations are as follows:

$$
\left\{\begin{array}{l}
\dot{v}_{x}=-\frac{1}{R_{1} C_{x}} v_{x}+\frac{1}{10 \cdot R_{2} C_{x}} v_{y} v_{z} \\
\dot{v}_{y}=-\frac{1}{R_{3} C_{y}} v_{y}+\frac{1}{R_{4} C_{y}}\left(\frac{R v_{u}}{R_{13}}+\frac{R}{100 R_{14}} v_{\varphi}^{2} v_{u}\right) \\
\dot{v}_{z}=\frac{1}{10 \cdot R_{5} C_{z}} v_{x} v_{y}-\frac{1}{R_{6} C_{z}} v_{z}+\frac{1}{R_{7} C_{z}} v_{w} \\
\dot{v}_{w}=\frac{1}{R_{8} C_{w}} v_{w}-\frac{1}{R_{9} C_{w}} v_{z} \\
\dot{v}_{u}=\frac{1}{R_{10} C_{u}} v_{u}-\frac{1}{100 \cdot R_{11} C_{u}} v_{x}^{2} v_{y} \\
\dot{v}_{\varphi}=\frac{1}{R_{12} C_{\varphi}} v_{u}
\end{array}\right.
$$

where $R_{1}=R / a, R_{3}=R / b, R_{4}=R / f, R_{6}=R / c, R_{7}=R / g$, $R_{8}=R / d, \quad R_{9}=R / h, \quad R_{10}=R / e, \quad R_{13}=R / f m, \quad$ and $R_{14}=R /(100 \cdot 3 f n)$. The hardware experiment simulation circuit of system (4) is shown in Figure 10. According to the parameter values in the four cases given in Table 2, the resistance values of the parameters in the equation are calculated when $C_{x}=C_{y}=C_{z}=C_{w}=C_{u}=C_{\varphi}=10 \mathrm{nF}$, $R=100 \mathrm{k} \Omega, \quad R_{2}=R_{5}=10 \mathrm{k} \Omega, \quad R_{11}=1 \mathrm{k} \Omega, \quad$ and $R_{12}=100 \mathrm{k} \Omega$. Figure 11 shows a group of phase portraits obtained by the Multisim simulator, which is basically consistent with the MATLAB numerical simulation results in the previous dynamic analysis and verifies the correctness of the chaotic circuit.

\section{Active Control Synchronization of the Novel 6D Memristive Hyperchaotic System}

At present, many synchronization methods are based on the synchronization between two identical systems, but between practical engineering applications, many systems are of different structures, so it is very important to realize the synchronization between two systems with different structures. The system mainly consists of two parts: one is the main system and the other is the slave system. This section mainly uses the method of active control to realize the synchronization of system (4). Set the main system as

$$
\left\{\begin{array}{l}
\dot{x}_{1}=a_{1} x_{1}+y_{1} z_{1}, \\
\dot{y}_{1}=-b_{1} y_{1}+f\left(m+3 n \varphi_{1}^{2}\right) l_{1}, \\
\dot{z}_{1}=-c_{1} z_{1}+x_{1} y_{1}+g_{1} w_{1}, \\
\dot{w}_{1}=d_{1} w_{1}-h_{1} z_{1}, \\
\dot{l}_{1}=e l_{1}-x_{1}^{2} y_{1}, \\
\dot{\varphi}_{1}=l_{1} .
\end{array}\right.
$$

The slave system is different from the main system in structure. The $6 \mathrm{D}$ hyperchaotic system designed by Yang et al. [46] is used as the slave system:

$$
\left\{\begin{array}{l}
\dot{x}_{2}=a_{2}\left(y_{2}-x_{2}\right)+w_{2}+u_{1}, \\
\dot{y}_{2}=c_{2} x_{2}-y_{2}-x_{2} z_{2}+l_{2}+u_{2}, \\
\dot{z}_{2}=-b_{2} z_{2}-x_{2} y_{2}+u_{3}, \\
\dot{w}_{2}=d_{2} w_{2}-x_{2} z_{2}+u_{4}, \\
\dot{l}_{2}=-k y_{2}+u_{5}, \\
\dot{\varphi}_{2}=h_{2} \varphi_{2}+u_{6},
\end{array}\right.
$$

where $u=\left[u_{1}, u_{2}, u_{3}, u_{4}, u_{5}, u_{6}\right]^{T}$ is the active controller of the synchronous system, which can make the main system and the slave system tend to be synchronous under different parameters and initial conditions. The error variable is made as shown in the following equation: 


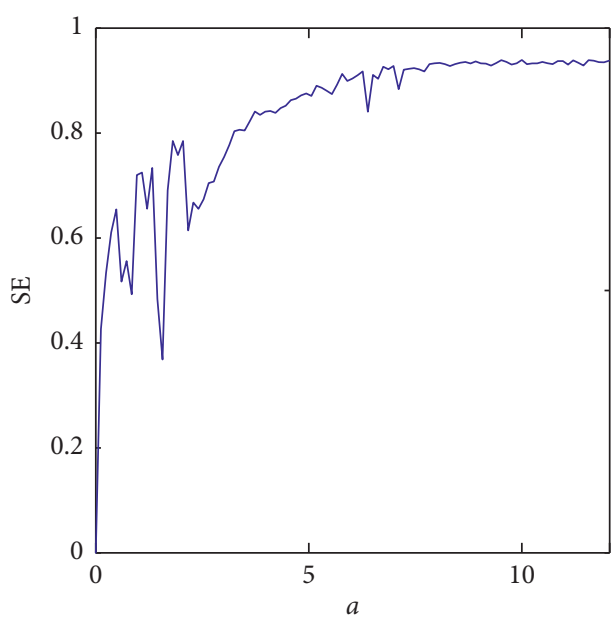

(a)

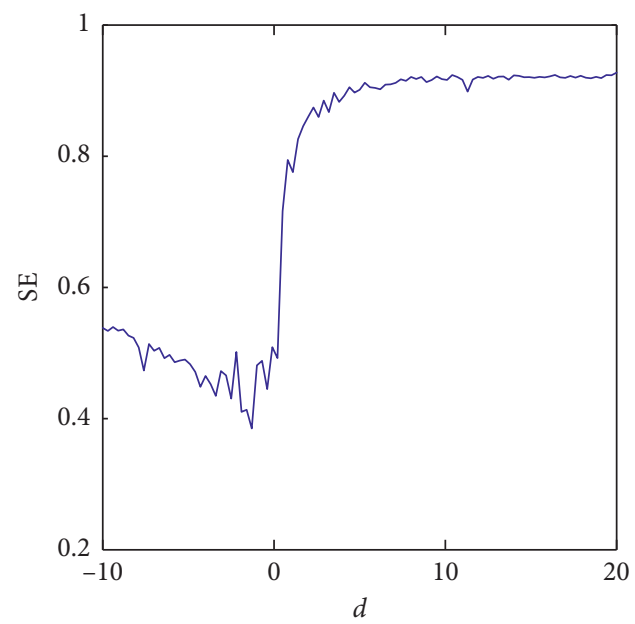

(b)

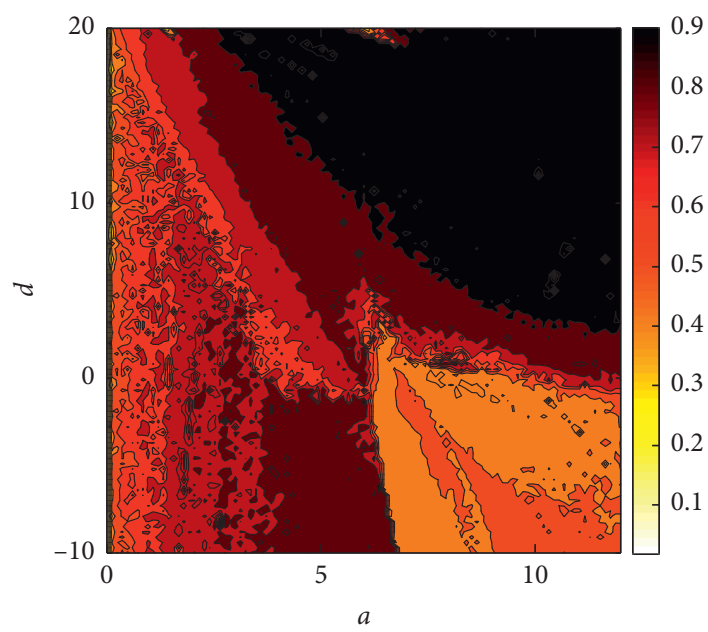

(c)

Figure 9: Spectral entropy (SE) complexity of system (4): (a) SE complexity versus $a(d=15)$; (b) SE complexity versus $d$ ( $a=10$ ); (c) SE complexity in the $a-d$ plane.

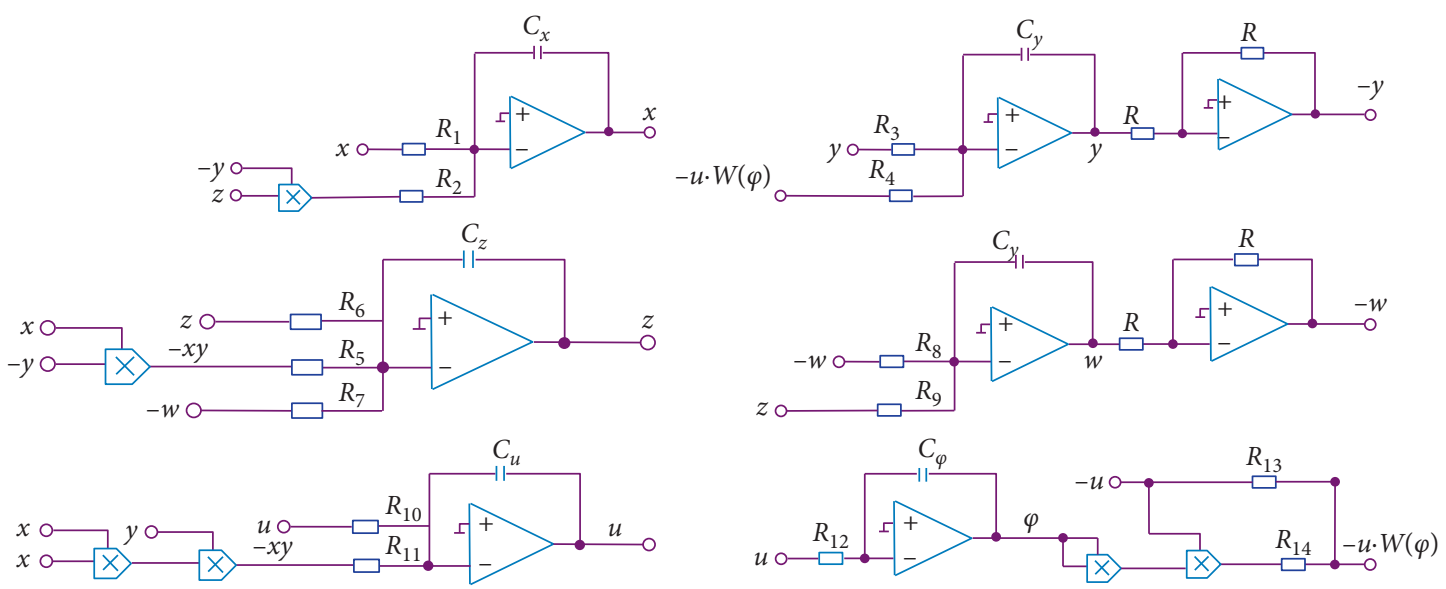

Figure 10: The circuit diagram of system (4). 
TABLE 2: Resistance values corresponding to different parameter values and simulation Figure 11.

\begin{tabular}{lclc}
\hline Case & \multicolumn{1}{c}{ Parameters } & Resistor & Figure \\
\hline Case & $a=1, b=8, c=1, d=-20, e=1$, & $R_{1}=100 \mathrm{k} \Omega, R_{3}=12.5 \mathrm{k} \Omega, R_{4}=50 \mathrm{k} \Omega, R_{6}=100 \mathrm{k} \Omega$, & Figure $11(\mathrm{a})$ \\
1 & $f=2, g=1, h=-1, m=1,3 n=0.02$ & $R_{7}=100 \mathrm{k} \Omega, R_{8}=5 \mathrm{k} \Omega, R_{9}=100 \mathrm{k} \Omega, R_{10}=100 \mathrm{k} \Omega$ & Figures $11(\mathrm{~b})$ \\
Case & $a=1, b=5, c=1, d=-20, e=1$, & $R_{1}=100 \mathrm{k} \Omega, R_{3}=20 \mathrm{k} \Omega, R_{4}=50 \mathrm{k} \Omega, R_{6}=100 \mathrm{k} \Omega$, & and $11(\mathrm{c})$ \\
2 & $f=2, g=1, h=-1, m=1,3 n=0.02$ & $R_{7}=100 \mathrm{k} \Omega, R_{8}=5 \mathrm{k} \Omega, R_{9}=100 \mathrm{k} \Omega, R_{10}=100 \mathrm{k} \Omega$ & Figures $11(\mathrm{~d})$ \\
Case & $a=2, b=6, c=1, d=-20, e=1$, & $R_{1}=50 \mathrm{k} \Omega, R_{3}=16.5 \mathrm{k} \Omega, R_{4}=50 \mathrm{k} \Omega, R_{6}=100 \mathrm{k} \Omega$, & and $11(\mathrm{e})$ \\
3 & $f=2, g=1, h=-1, m=1,3 n=0.02$ & $R_{7}=100 \mathrm{k} \Omega, R_{8}=5 \mathrm{k} \Omega, R_{9}=100 \mathrm{k} \Omega, R_{10}=100 \mathrm{k} \Omega$ & Figure $11(\mathrm{f})$ \\
Case & $a=2, b=6, c=2, d=-2, e=2$, & $R_{1}=50 \mathrm{k} \Omega, R_{3}=16.5 \mathrm{k} \Omega, R_{4}=50 \mathrm{k} \Omega, R_{6}=50 \mathrm{k} \Omega$, & \\
4 & $f=2, g=-1, h=-1, m=1,3 n=0.02$ & $R_{7}=100 \mathrm{k} \Omega, R_{8}=50 \mathrm{k} \Omega, R_{9}=100 \mathrm{k} \Omega, R_{10}=50 \mathrm{k} \Omega$ & \\
\hline
\end{tabular}

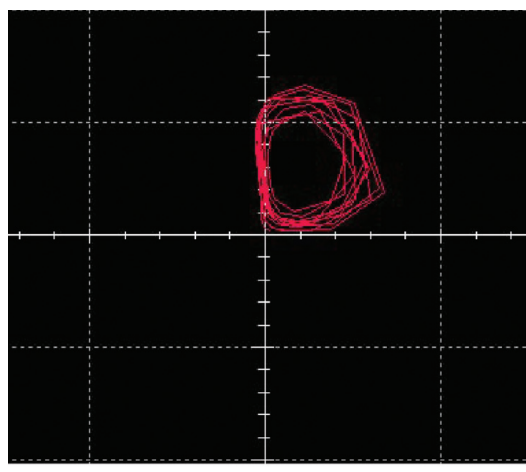

(a)

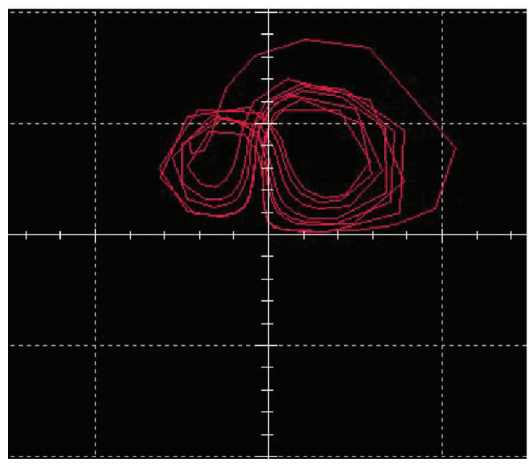

(d)

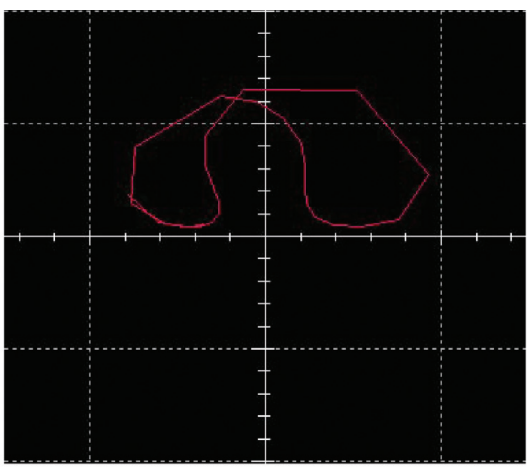

(b)

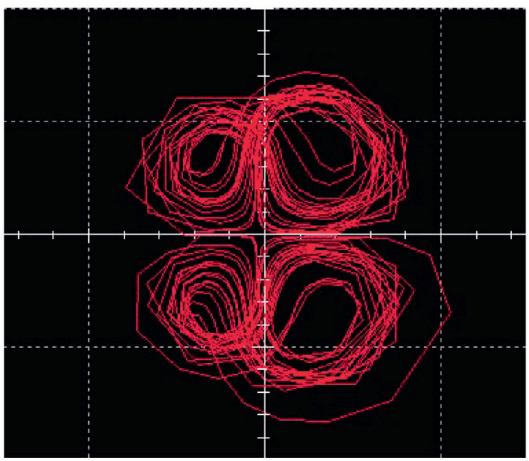

(e)

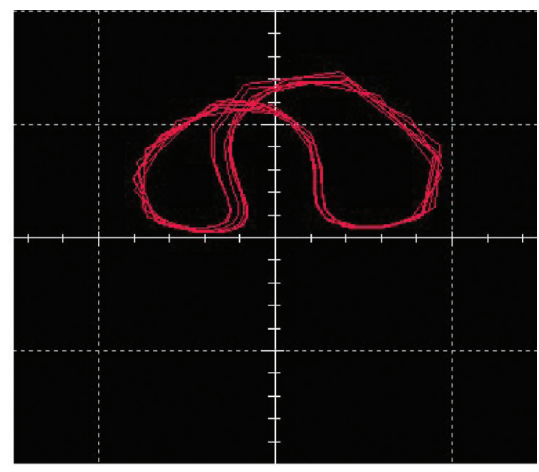

(c)

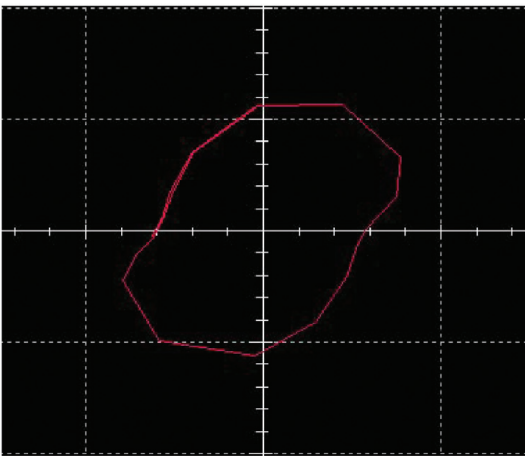

(f)

FIGURE 11: The circuit simulation diagram of system (4): (a) one-wing multiperiod, (b) period-1, (c) two-wing multiperiod, (d) two-wing, (e) four-wing, and (f) limit cycle.

$$
\left\{\begin{array}{l}
e_{1}=x_{2}-x_{1}, \\
e_{2}=y_{2}-y_{1}, \\
e_{3}=z_{2}-z_{1}, \\
e_{4}=w_{2}-w_{1}, \\
e_{5}=l_{2}-l_{1}, \\
e_{6}=\varphi_{2}-\varphi_{1} .
\end{array}\right.
$$

Therefore, from the error variable, the main system (16), and the slave system (17), the error system equation can be obtained: 


$$
\left\{\begin{array}{l}
\dot{e}_{1}=a_{2} e_{2}-\left(a_{2}+a_{1}\right) e_{1}+e_{4}+a_{2} y_{1}-a_{2} x_{1}+a_{1} x_{2}-y_{1} z_{1}+w_{1}+u_{1} \\
\dot{e}_{2}=c_{2} e_{1}+c_{2} x_{1}-\left(1+b_{1}\right) e_{2}-y_{1}-x_{2} z_{2}+(1+f m) e_{5}+b_{1} y_{2}-f m l_{2}-3 n f \varphi_{1}^{2} l_{1}+l_{1}+u_{2} \\
\dot{e}_{3}=\left(-b_{2}-c_{1}\right) e_{3}-b_{2} z_{1}+x_{2} y_{2}+c_{1} z_{2}-x_{1} y_{1}+g_{1} e_{4}-g_{1} w_{2}+u_{3} \\
\dot{e}_{4}=\left(d_{2}+d_{1}\right) e_{4}+d_{2} w_{1}-h_{1} e_{3}-x_{2} z_{2}-d_{1} w_{2}+h_{1} z_{2}+u_{4} \\
\dot{e}_{5}=-k e_{2}+e e_{5}-k y_{1}-e_{2}+x_{1}^{2} y_{1}+u_{5} \\
\dot{e}_{6}=h_{2} e_{6}+g_{2} e_{2}+e_{5}+h_{2} \varphi_{1}+g_{2} y_{1}+u_{6}
\end{array}\right.
$$

By simplifying the linear term of equation (19), the active control function is obtained:

$$
\left\{\begin{array}{l}
u_{1}=-a_{2} y_{1}+a_{2} x_{1}-a_{1} x_{2}+y_{1} z_{1}-w_{1}+v_{1} \\
u_{2}=-c_{2} x_{1}+y_{1}+x_{2} z_{2}-b_{1} y_{2}+f m l_{2}+3 n f \varphi_{1}^{2} l_{1}-l_{1}+v_{2} \\
u_{3}=b_{2} z_{1}-x_{2} y_{2}-c_{1} z_{2}+x_{1} y_{1}+g_{1} w_{2}+v_{3} \\
u_{4}=d_{2} w_{1}+x_{2} z_{2}+d_{1} w_{2}-h_{1} z_{2}+v_{4} \\
u_{5}=k y_{1}+e l_{2}-x_{1}^{2} y_{1}+v_{5} \\
u_{6}=-h_{2} \varphi_{1}-g_{2} y_{1}+v_{6}
\end{array}\right.
$$

where $v=\left[v_{1}, v_{2}, v_{3}, v_{4}, v_{5}, v_{6}\right]^{T}$ is the control input, and the linear error system without an active controller can be obtained by taking (20) into (19):

$$
\left\{\begin{array}{l}
\dot{e}_{1}=a_{2} e_{2}-\left(a_{2}+a_{1}\right) e_{1}+e_{4}+v_{1} \\
\dot{e}_{2}=c_{2} e_{1}+(1+f m) e_{5}-\left(1+b_{1}\right) e_{2}+v_{2} \\
\dot{e}_{3}=g_{1} e_{4}+\left(-b_{2}-c_{1}\right) e_{3}+v_{3} \\
\dot{e}_{4}=\left(d_{2}+d_{1}\right) e_{4}-h_{1} e_{3}+v_{4} \\
\dot{e}_{5}=-k e_{2}+e e_{5}+v_{5} \\
\dot{e}_{6}=h_{2} e_{6}+g_{2} e_{2}+e_{5}+v_{6}
\end{array}\right.
$$

To synchronize the system, we need to

$$
\lim _{x \longrightarrow \infty} e_{i}=0, \quad(i=1,2,3,4,5,6) .
$$

The above formula shows that if system (21) tends to be stable with time and under the control input $v=\left[v_{1}, v_{2}, v_{3}, v_{4}, v_{5}, v_{6}\right]^{T}$, then the error variable $e=\left[e_{1}, e_{2}, e_{3}, e_{4}, e_{5}, e_{6}\right]^{T}$ tends to zero and then the main system (16) and the slave system (17) are synchronized. To achieve this goal, we define a matrix $A$ to express the relationship between the error system and the control input, which can be expressed as

$$
v=A \cdot e .
$$

According to the criteria of Routh-Hurwitz, if equation (19) is stable, all eigenvalues of a matrix must be negative. Therefore, equation (19) can be expressed as

$$
\left[\begin{array}{l}
v_{1} \\
v_{2} \\
v_{3} \\
v_{4} \\
v_{5} \\
v_{6}
\end{array}\right]=\left[\begin{array}{cccccc}
a_{1}+a_{2}-1 & -a_{2} & 0 & 1 & 0 & 0 \\
-c_{2} & b_{1} & 0 & 0 & -f m-1 & 0 \\
0 & 0 & b_{2}+c_{1}-1 & 0 & 0 & 0 \\
0 & 0 & h_{1} & -d_{1}-d_{2}-1 & 0 & 0 \\
0 & k & 0 & 0 & -e-1 & 0 \\
0 & -g_{2} & 0 & 0 & -1 & -h_{2}-1
\end{array}\right]\left[\begin{array}{l}
e_{1} \\
e_{2} \\
e_{3} \\
e_{4} \\
e_{5} \\
e_{6}
\end{array}\right]
$$

Then, the eigenvalue of the error system (21) is $-1,-1$, $-1,-1,-1$, and -1 , so equation (24) can be reduced to

$$
\left\{\begin{array}{l}
v_{1}=\left(a_{1}+a_{2}-1\right)\left(x_{2}-x_{1}\right)+\left(w_{2}-w_{1}\right)-a_{2}\left(y_{2}-y_{1}\right), \\
v_{2}=-c_{2}\left(x_{2}-x_{1}\right)+b_{1}\left(y_{2}-y_{1}\right)+(-f m-1)\left(l_{2}-l_{1}\right), \\
v_{3}=\left(b_{2}+c_{1}-1\right)\left(z_{2}-z_{1}\right) \\
v_{4}=h_{1}\left(z_{2}-z_{1}\right)+\left(-d_{1}-d_{2}-1\right)\left(w_{2}-w_{1}\right), \\
v_{5}=k\left(y_{2}-y_{1}\right)+(-e-1)\left(l_{2}-l_{1}\right) \\
v_{6}=-g_{2}\left(y_{2}-y_{1}\right)-\left(l_{2}-l_{1}\right)+\left(-h_{2}-1\right)\left(\varphi_{2}-\varphi_{1}\right) .
\end{array}\right.
$$



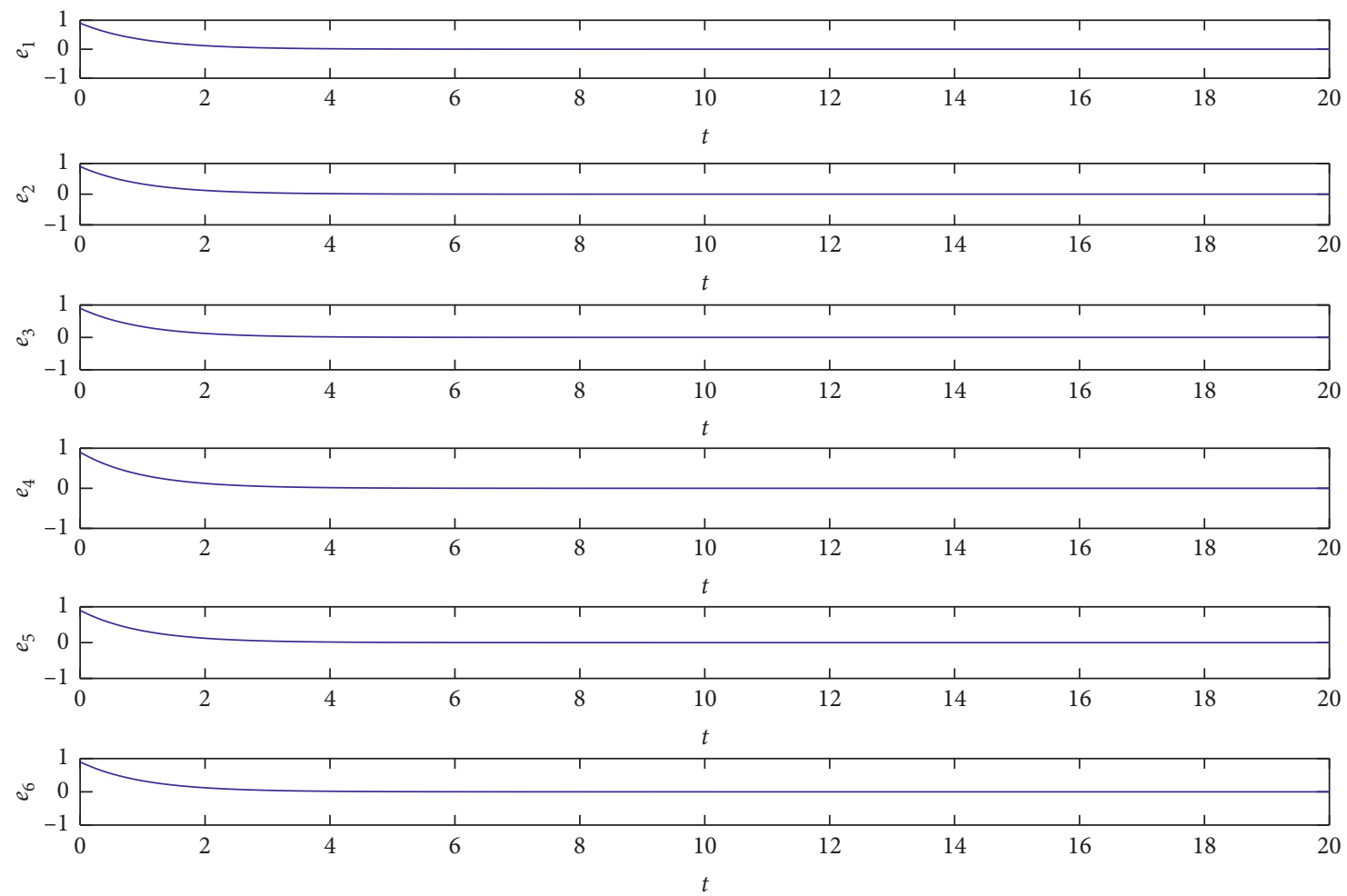

FIGURE 12: The trajectories of the synchronization errors $e_{1}, e_{2}, e_{3}, e_{4}, e_{5}$, and $e_{6}$.

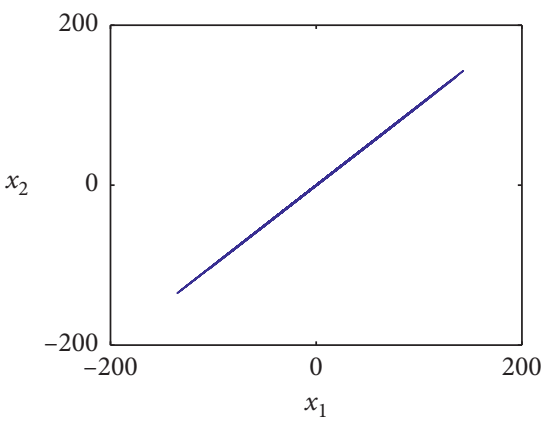

(a)

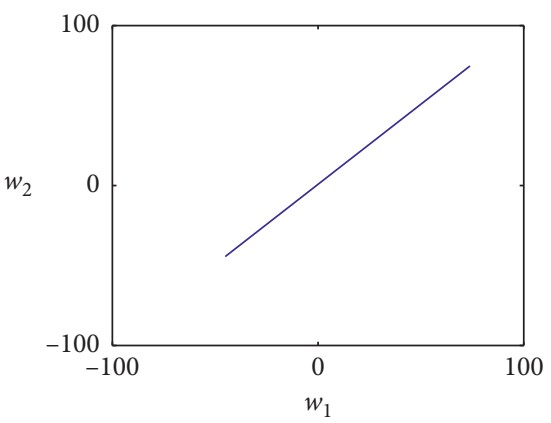

(d)

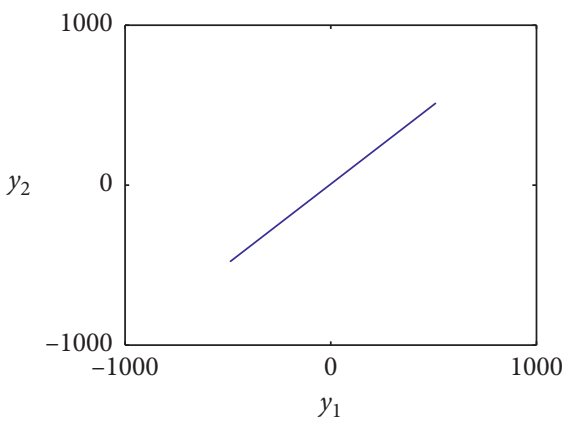

(b)

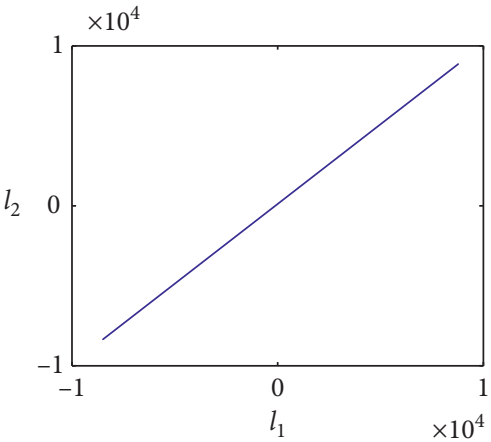

(e)

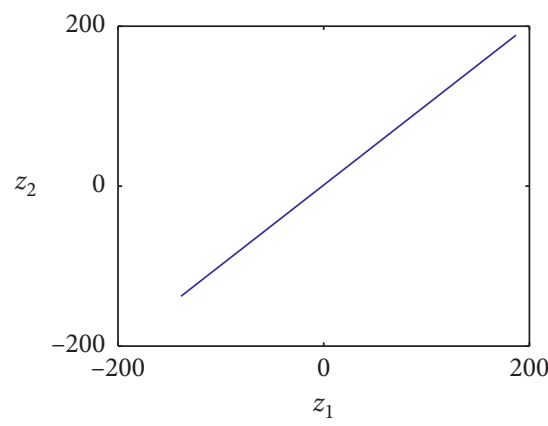

(c)

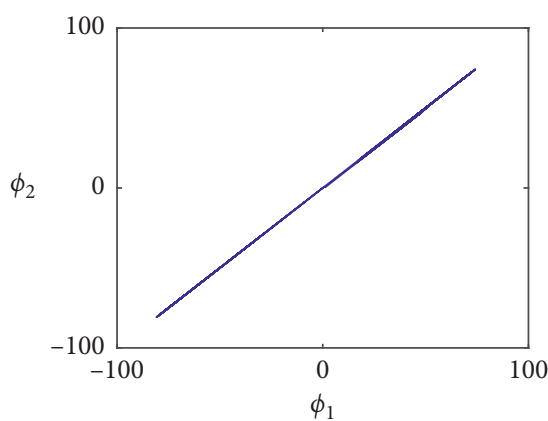

(f)

FiguRe 13: Synchronous phase diagram of two different structure systems in the corresponding plane. (a) $x_{1}-x_{2}$, (b) $y_{1}-y_{2}$, (c) $z_{1}-z_{2}$, (d) $w_{1}-w_{2}$, (e) $l_{1}-l_{2}$, and (f) $\varphi_{1}-\varphi_{2}$. 
The main slave system is simulated by MATLAB to verify whether the proposed system can achieve synchronization. According to the system equation, the parameters of the main system (16) are given as $a_{1}=10, b_{1}=60, c_{1}=20, d_{1}=$ $15, e=40, f=1, g_{1}=50, h_{1}=10, m=1$, and $3 n=0.02$, the parameters of the slave system (17) are set as $a_{2}=10, b_{2}=$ $8 / 3, c_{2}=28, d_{2}=2, g_{2}=1, k=8.4$, and $h_{2}=1$, and the initial conditions of the main slave system are set as $[1,1,1,1,1,1]$ and $[0.1,0.1,0.1,0.1,0.1,0.1]$, respectively. Figure 12 shows a simulation diagram of the system error. It can be seen from Figure 12 that when $t>2$, two different structure hyperchaotic systems realize global synchronization. From Figure 13, it can also be seen from the six phase planes that the two systems realize synchronization.

\section{Conclusion}

This work presents a novel 6D memristive four-wing hyperchaotic system. Dynamical analysis and numerical simulation of the novel chaotic system were first carried out. Further analysis of the novel system shows that the multiple coexisting attractors can be observed with different system parameter values and initial values. Then, circuitry of the novel chaotic system was designed. The numerical and electronic circuit simulation results were found to be in good accordance. Besides, synchronization between the proposed 6D memristive hyperchaotic system and the 6D hyperchaotic Yang system with different structures was realized by an active control approach for secure communication applications, and the accuracy and validity of the results were verified by theoretical analysis and numerical simulations.

\section{Data Availability}

All data used to support the findings of this study are available from the corresponding author upon request.

\section{Conflicts of Interest}

The authors declare that they have no conflicts of interest.

\section{Acknowledgments}

This work was supported by the National Natural Science Foundation of China under grants 61504013, 61702052, $61772087,61741104,61674054$, and 61901169, by the Natural Science Foundation of Hunan Province under grants 2019JJ50648, 2016jj2005, 2017JJ2049, and 2019JJ40190, by the Scientific Research Fund of Hunan Provincial Education Department under grants 19C0083 and 18A137, by the young teacher development program project of Changsha University of Science and Technology under grant 2019QJCZ013, by the National Key Research and Development Project under grant 2018YFE0111200, by the Guizhou Provincial Science and Technology Foundation under grant [2018]1115, and by the Guizhou Province Science and Technology Plan Project under grant [2018] 5769.

\section{References}

[1] A. Dmitriev, V. Kornilov, and S. Maltseva, "Complexity of a microblogging social network in the framework of modern nonlinear science," Complexity, vol. 2018, Article ID 4732491, 11 pages, 2018.

[2] X. Zhang, C. Wang, W. Yao, and H. Lin, "Chaotic system with bondorbital attractors," Nonlinear Dynamics, vol. 97, no. 4, pp. 2159-2174, 2019.

[3] F. Wang, L. Zhang, S. Zhou, and Y. Huang, "Neural networkbased finite-time control of quantized stochastic nonlinear systems,” Neurocomputing, vol. 362, pp. 195-202, 2019.

[4] F. Yu, L. Liu, L. Xiao, K. Li, and S. Cai, "A robust and fixedtime zeroing neural dynamics for computing time-variant nonlinear equation using a novel nonlinear activation function," Neurocomputing, vol. 350, pp. 108-116, 2019.

[5] J. Jin, L. Zhao, M. Li, F. Yu, and Z. Xi, "Improved zeroing neural networks for finite time solving nonlinear equations," Neural Computing and Applications, vol. 32, no. 9, pp. 4151-4160, 2020.

[6] F. Tan, L. Zhou, F. Yu, and J. Lu, "Fixed-time continuous stochastic synchronisation of two-layer dynamical networks," International Journal of Systems Science, vol. 51, no. 2, pp. 242-257, 2020.

[7] M. Long, F. Peng, and Y. Zhu, "Identifying natural images and computer generated graphics based on binary similarity measures of PRNU," Multimedia Tools and Applications, vol. 78, no. 1, pp. 489-506, 2019.

[8] L. Zhou, F. Tan, F. Yu, and W. Liu, "Cluster synchronization of two-layer nonlinearly coupled multiplex networks with multi-links and time-delays," Neurocomputing, vol. 359, pp. 264-275, 2019.

[9] W. Yao, C. Wang, J. Cao, Y. Sun, and C. Zhou, "Hybrid multisynchronization of coupled multistable memristive neural networks with time delays," Neurocomputing, vol. 363, pp. 281-294, 2019.

[10] H. Lin and C. Wang, "Influences of electromagnetic radiation distribution on chaotic dynamics of a neural network," Applied Mathematics and Computation, vol. 369, Article ID 124840, 2020.

[11] L. Zhou and F. Tan, "A chaotic secure communication scheme based on synchronization of double-layered and multiple complex networks," Nonlinear Dynamics, vol. 96, no. 2, pp. 869-883, 2019.

[12] X. Zhang and C. Wang, "A novel multi-attractor period multiscroll chaotic integrated circuit based on CMOS wide adjustable CCCII," IEEE Access, vol. 7, no. 1, pp. 16336-16350, 2019.

[13] J. Jin and L. Zhao, "Low voltage low power fully integrated chaos generator," Journal of Circuits Systems and Computers, vol. 27, no. 10, Article ID 1850155, 2018.

[14] J. Jin, "Programmable multi-direction fully integrated chaotic oscillator," Microelectronics Journal, vol. 75, pp. 27-34, 2018.

[15] F. Yu, H. Shen, L. Liu et al., "CCII and FPGA Realization: a multistable modified four-order autonomous Chua's chaotic system with coexisting multiple attractors," Complexity, vol. 2020, Article ID 5212601, 17 pages, 2020.

[16] Q. Yin and C. H. Wang, "A new chaotic image encryption scheme using breadth-first search and dynamic diffusion," International Journal of Bifurcation and Chaos, vol. 28, no. 4, Article ID 1850047, 2018.

[17] S. Wang, C. Wang, and C. Xu, "An image encryption algorithm based on a hidden attractor chaos system and the Knuth-Durstenfeld algorithm," Optics and Lasers in Engineering, vol. 128, Article ID 105995, 2020. 
[18] G. Cheng, C. Wang, and H. Chen, "A novel color image encryption algorithm based on hyperchaotic system and permutation-diffusion architecture," International Journal of Bifurcation and Chaos, vol. 29, no. 9, Article ID 1950115, 2019.

[19] M. Long, F. Peng, and H.-y. Li, "Separable reversible data hiding and encryption for HEVC video," Journal of Real-Time Image Processing, vol. 14, no. 1, pp. 171-182, 2018.

[20] Y. Chen, J. Wang, R. Xia, Q. Zhang, Z. Cao, and K. Yang, "The visual object tracking algorithm research based on adaptive combination kernel," Journal of Ambient Intelligence and Humanized Computing, vol. 10, no. 12, pp. 4855-4867, 2019.

[21] F. Yu, Q. Wan, J. Jin et al., "Design and FPGA implementation of a pseudorandom number generator based on a four-wing memristive hyperchaotic system and Bernoulli map," IEEE Access, vol. 7, pp. 181884-181898, 2019.

[22] F. Yu, L. Li, Q. Tang, S. Cai, Y. Song, and Q. Xu, "A survey on true random number generators based on chaos," Discrete Dynamics in Nature and Society, vol. 2019, Article ID 2545123, 10 pages, 2019.

[23] F. Yu, L. Liu, S. Qian et al., "Chaos-based application of a novel multistable 5D memristive hyperchaotic system with coexisting multiple attractors," Complexity, vol. 2020, Article ID 8034196, 19 pages, 2020.

[24] F. Yu and C. Wang, "Secure communication based on a fourwing chaotic system subject to disturbance inputs," Optik, vol. 125 , no. 20 , pp. 5920-5925, 2014.

[25] L. L. Zhou, F. Tan, and F. Yu, "A robust synchronizationbased chaotic secure communication scheme with doublelayered and multiple hybrid networks," IEEE Systems Journal, pp. 1-12, 2019.

[26] F. Yu, L. Gao, K. Gu, B. Yin, Q. Wan, and Z. Zhou, "A fully qualified four-wing four-dimensional autonomous chaotic system and its synchronization," Optik, vol. 131, pp. 79-88, 2017.

[27] F. Yu, C. H. Wang, J. W. Yin et al., "Novel four-dimensional autonomous chaotic system generating one-, two-, three-and four-wing attractors," Chinese Physics B, vol. 20, no. 11, Article ID 110505, 2011.

[28] L. Zhou, C. Wang, and L. Zhou, "A novel no-equilibrium hyperchaotic multi-wing system via introducing memristor," International Journal of Circuit Theory and Applications, vol. 46, no. 1, pp. 84-98, 2018.

[29] Q. Deng and C. Wang, "Multi-scroll hidden attractors with two stable equilibrium points," Chaos: An Interdisciplinary Journal of Nonlinear Science, vol. 29, no. 9, Article ID 093112, 2019.

[30] F. Yu, P. Li, K. Gu, and B. Yin, "Research progress of multiscroll chaotic oscillators based on current-mode devices," Optik, vol. 127, no. 13, pp. 5486-5490, 2016.

[31] C. Wang, X. Liu, and H. Xia, "Multi-piecewise quadratic nonlinearity memristor and its $2 \mathrm{~N}$-scroll and $2 \mathrm{~N}+1$-scroll chaotic attractors system," Chaos: An Interdisciplinary Journal of Nonlinear Science, vol. 27, no. 3, Article ID 033114, 2017.

[32] F. Yu, C. Wang, and H. He, "Grid multiscroll hyperchaotic attractors based on colpitts oscillator mode with controllable grid gradient and scroll numbers," Journal of Applied Research and Technology, vol. 11, no. 3, pp. 371-380, 2013.

[33] A. Lassoued and O. Boubaker, "Dynamic analysis and circuit design of a novel hyperchaotic system with fractional-order terms," Complexity, vol. 2017, Article ID 3273408, 10 pages, 2017.

[34] A. G. Radwan, A. T. Azar, S. Vaidyanathan, J. M. MunozPacheco, and A. Ouannas, "Fractional-order and memristive nonlinear systems: advances and applications," Complexity, vol. 2017, Article ID 3760121, 2 pages, 2017.

[35] X. Zhang and Z. Li, "Hidden extreme multistability in a novel $4 \mathrm{D}$ fractional-order chaotic system," International Journal of Non-linear Mechanics, vol. 111, pp. 14-27, 2019.

[36] O. E. Rossler, "An equation for hyperchaos," Physics Letters A, vol. 71, no. 2-3, pp. 155-157, 1979.

[37] R. Barboza, "Dynamics of a hyperchaotic lorenz system," International Journal of Bifurcation and Chaos, vol. 17, no. 12, pp. 4285-4294, 2011.

[38] X. J. Wu and X. Y. Wang, "Chaos synchronization of a new hyperchaotic Chen system via nonlinear control," Acta Physica Sinica, vol. 55, no. 12, pp. 6261-6266, 2006.

[39] G. Wang, X. Zhang, Y. Zheng, and Y. Li, "A new modified hyperchaotic Lü system,” Physica A: Statistical Mechanics and its Applications, vol. 371, no. 2, pp. 260-272, 2006.

[40] F. Yu, C. H. Wang, Y. Hu, and J. W. Yin, "Antisynchronization of a novel hyperchaotic system with parameter mismatch and external disturbances," Pramana, vol. 79, no. 1, pp. 81-93, 2012.

[41] H. Wang and G. Dong, "New dynamics coined in a 4-D quadratic autonomous hyper-chaotic system," Applied Mathematics and Computation, vol. 346, no. 1, pp. 272-286, 2019.

[42] S. Vaidyanathan, "A ten-term novel 4-D hyperchaotic system with three quadratic nonlinearities and its control," International Journal of Control Theory and Applications, vol. 6, pp. 97-109, 2013.

[43] S. Vaidyanathan, C. Volos, and V.-T. Pham, "Hyperchaos, adaptive control and synchronization of a novel 5-D hyperchaotic system with three positive Lyapunov exponents and its SPICE implementation," Archives of Control Sciences, vol. 24, no. 4, pp. 409-446, 2014.

[44] K. Kemih, M. Ghanes, R. Remmouche, and A. Senouci, "A novel 5D-dimentional hyperchaotic system and its circuit simulation by EWB," Mathematical Sciences Letters, vol. 4, no. 1, pp. 1-4, 2015.

[45] M. M. Aziz and S. F. AL-Azzawi, "Chaos control and synchronization of a novel 5-D hyperchaotic lorenz system via nonlinear control," International Journal of Modern Physics and Application, vol. 2, no. 6, pp. 110-115, 2015.

[46] Q. Yang, W. M. Osman, and C. Chen, "A new 6D hyperchaotic system with four positive lyapunov exponents coined," International Journal of Bifurcation and Chaos, vol. 25, no. 4, Article ID 1550060, 2015.

[47] S. Cang, G. Qi, and Z. Chen, "A four-wing hyper-chaotic attractor and transient chaos generated from a new 4-D quadratic autonomous system," Nonlinear Dynamics, vol. 59, no. 3, pp. 515-527, 2010.

[48] A. Zarei, "Complex dynamics in a 5-D hyper-chaotic attractor with four-wing, one equilibrium and multiple chaotic attractors," Nonlinear Dynamics, vol. 81, no. 1-2, pp. 585-605, 2015.

[49] J. Jin and L. Cui, "Fully integrated memristor and its application on the scroll-controllable hyperchaotic system," Complexity, vol. 2019, Article ID 4106398, 2019.

[50] Q. Zhao, C. H. Wang, and X. Zhang, "A universal emulator for memristor, memcapacitor, and meminductor and its chaotic circuit," Chaos: An Interdisciplinary Journal of Nonlinear Science, vol. 29, no. 1, Article ID 013141, 2019.

[51] V. T. Pham, S. Vaidyanathan, E. Tlelo-Cuautle et al., "Memory circuit elements: complexity, complex systems, and applications," Complexity, vol. 2019, Article ID 4936123, 4 pages, 2019. 
[52] D. B. Strukov, G. S. Snider, D. R. Stewart, and R. S. Williams, "The missing memristor found," Nature, vol. 453, no. 7191, pp. 80-83, 2008.

[53] L. Chua, "Memristor-the missing circuit element," IEEE Transactions on Circuit Theory, vol. 18, no. 5, pp. 507-519, 1971.

[54] M. Itoh and L. O. Chua, "Memristor oscillators," International Journal of Bifurcation and Chaos, vol. 18, no. 11, pp. 31833206, 2008.

[55] J.-Y. Ruan, K.-H. Sun, and J. Mou, "Memristor-based Lorenz hyper-chaotic system and its circuit implementation," Acta Physica Sinica, vol. 65, no. 19, Article ID 190502, 2016.

[56] L. Zhou, C. Wang, and L. Zhou, "Generating hyperchaotic multi-wing attractor in a 4D memristive circuit," Nonlinear Dynamics, vol. 85, no. 4, pp. 2653-2663, 2016.

[57] L. Zhou, C. Wang, and L. Zhou, "Generating four-wing hyperchaotic attractor and two-wing, three-wing, and fourwing chaotic attractors in 4D memristive system," International Journal of Bifurcation and Chaos, vol. 27, no. 2, Article ID 1750027, 2017.

[58] C. Wang, H. Xia, and L. Zhou, "A memristive hyperchaotic multiscroll Jerk system with controllable scroll numbers," International Journal of Bifurcation and Chaos, vol. 27, no. 6, Article ID 1750091, 2017.

[59] C. Wang, L. Zhou, and R. Wu, "The design and realization of a hyper-chaotic circuit based on a flux-controlled memristor with linear memductance," Journal of Circuits, Systems and Computers, vol. 27, no. 3, Article ID 1850038, 2018.

[60] F. Yu, L. Liu, B. He et al., "Analysis and FPGA realization of a novel 5D hyperchaotic four-wing memristive system, active control synchronization and secure communication application," Complexity, vol. 2019, Article ID 4047957, 18 pages, 2019.

[61] F. Yu, Z. Zhang, L. Liu et al., "Secure communication scheme based on a new 5D multistable four-wing memristive hyperchaotic system with disturbance inputs," Complexity, vol. 2020, Article ID 5859273, 16 pages, 2020.

[62] B. A. Mezatio, M. T. Motchongom, B. R. Wafo Tekam, R. Kengne, R. Tchitnga, and A. Fomethe, "A novel memristive 6D hyperchaotic autonomous system with hidden extreme multistability," Chaos, Solitons and Fractals, vol. 120, pp. 100-115, 2019.

[63] W. Li, Z. Chen, X. Gao, W. Liu, and J. Wang, "MultiModel framework for indoor localization under mobile edge computing environment," IEEE Internet of Things Journal, vol. 6, no. 3, pp. 4844-4853, 2019.

[64] K. Gu, X. Dong, and L. Wang, "Efficient traceable ring signature scheme without pairings," Advances in Mathematics of Communications, vol. 14, no. 2, pp. 207-232, 2020.

[65] W. Li, Y. Ding, Y. Yang et al., "Parameterized algorithms of fundamental NP-hard problems: a survey," Human-Centric Computing and Information Sciences, 2020.

[66] Y. Chen, W. Xu, J. Zuo, and K. Yang, "The fire recognition algorithm using dynamic feature fusion and IV-SVM classifier," Cluster Computing, vol. 22, no. S3, pp. 7665-7675, 2019.

[67] K. Gu, K. Wang, and L. Yang, "Traceable attribute-based signature," Journal of Information Security and Applications, vol. 49, pp. 102400-102416, 2019.

[68] L. Xiang, G. Guo, J. Yu, V. S. Sheng, and P. Yang, “A convolutional neural network-based linguistic steganalysis for synonym substitution steganography," Mathematical Biosciences and Engineering, vol. 17, no. 2, pp. 1041-1058, 2020.
[69] W. Li, H. Xu, H. Li et al., "Complexity and algorithms for superposed data uploading problem in networks with smart devices," IEEE Internet of Things Journal, p. 1, 2019.

[70] K. Gu, N. Wu, B. Yin, and W. Jia, "Secure data query framework for cloud and fog computing," IEEE Transactions on Network and Service Management, vol. 17, no. 1, pp. 332-345, 2020.

[71] Y. Chen, J. Wang, X. Chen et al., "Single-image super-resolution algorithm based on structural self-similarity and deformation block features," IEEE Access, vol. 7, pp. 58791-58801, 2019.

[72] K. Gu, W. Zhang, S.-J. Lim, P. K. Sharma, Z. Al-Makhadmeh, and A. Tolba, "Reusable mesh signature scheme for protecting identity privacy of IoT devices," Sensors, vol. 20, no. 3, p. 758, 2020.

[73] L. M. Pecora and T. L. Carroll, "Synchronization in chaotic systems,” Physical Review Letters, vol. 64, no. 8, pp. 821-824, 1990.

[74] G. M. Mahmoud and E. E. Mahmoud, "Complete synchronization of chaotic complex nonlinear systems with uncertain parameters," Nonlinear Dynamics, vol. 62, no. 4, pp. 875-882, 2010.

[75] W. S. Sayed, M. M. R. Henein, S. K. Abd-El-Hafiz, and A. G. Radwan, "Generalized dynamic switched synchronization between combinations of fractional-order chaotic systems," Complexity, vol. 2017, Article ID 9189120, 17 pages, 2017.

[76] F. Yu, C.-H. Wang, Y. Hu, and J.-W. Yin, "Projective synchronization of a five-term hyperbolic-type chaotic system with fully uncertain parameters," Acta Physica Sinica, vol. 61, no. 6, Article ID 060505, 2012.

[77] C.-H. Wang, Y. Hu, F. Yu, and H. Xu, "Time-controllable projective synchronization of a class of chaotic systems based on adaptive method," Acta Physica Sinica, vol. 62, no. 11, Article ID 110509, 2013.

[78] X.-T. Tran and H.-J. Kang, "Fixed-time complex modified function projective lag synchronization of chaotic (hyperchaotic) complex systems," Complexity, vol. 2017, Article ID 4020548, 9 pages, 2017.

[79] F. Yu and Y. Song, "Complete switched generalized function projective synchronization of a class of hyperchaotic systems with unknown parameters and disturbance inputs," Journal of Dynamic Systems, Measurement, and Control-Transactions of the ASME, vol. 136, no. 1, Article ID 014505, 2014.

[80] F. Yu, S. Qian, X. Chen et al., "A new 4D four-wing memristive hyperchaotic system: dynamical analysis, electronic circuit design, shape synchronization and secure communication," International Journal of Bifurcation and Chaos, 2020.

[81] Y. Peng, K. Sun, D. Peng, and W. Ai, "Dynamics of a higher dimensional fractional-order chaotic map," Physica A: Statistical Mechanics and its Applications, vol. 525, pp. 96107, 2019.

[82] S. He, N. A. A. Fataf, S. Banerjee, and K. Sun, "Complexity in the muscular blood vessel model with variable fractional derivative and external disturbances," Physica A: Statistical Mechanics and its Applications, vol. 526, Article ID 120904, 2019.

[83] F. Yu, L. Gao, L. Liu, S. Qian, S. Cai, and Y. Song, "A 1 V, 0.53 ns, $59 \mu \mathrm{W}$ current comparator using standard $0.18 \mu \mathrm{m}$ CMOS technology," Wireless Personal Communications, vol. 111, no. 2, pp. 843-851, 2020. 
[84] F. Yu, "A low-voltage and low-power 3-GHz CMOS LC VCO for S-band wireless applications," Wireless Personal Communications, vol. 78, no. 2, pp. 905-914, 2014.

[85] F. Yu, Q. Tang, W. Wang, and H. Wu, “A 2.7 GHz low-phasenoise LC-QVCO using the gate-modulated coupling technique," Wireless Personal Communications, vol. 86, no. 2, pp. 671-681, 2016. 